\title{
Atomistic modelling of electrocatalysis: Are we there yet?
}

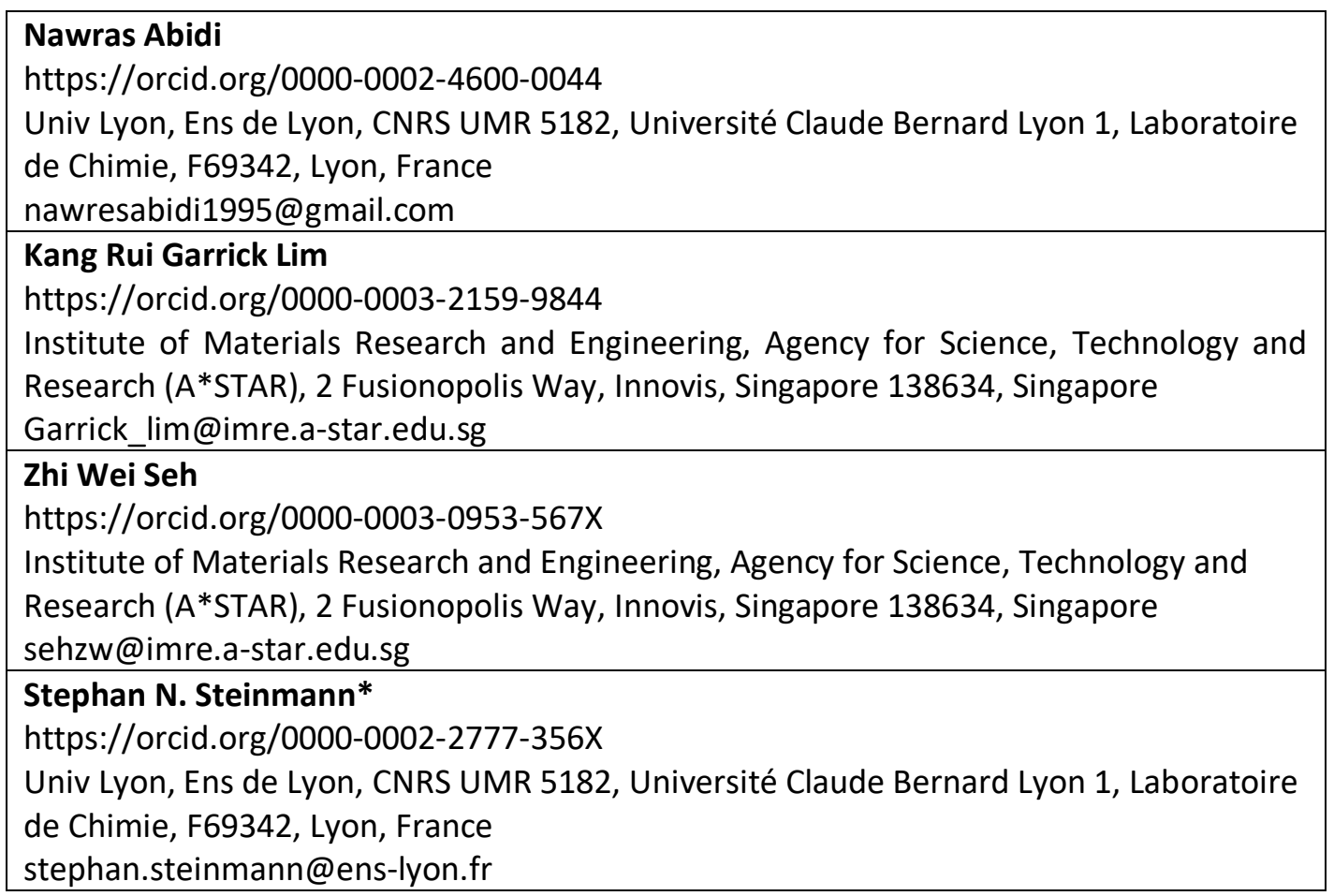

\section{Abstract}

Electrified interfaces play a prime role in energy technologies, from batteries and capacitors to heterogeneous electrocatalysis. The atomistic understanding and modelling of these interfaces is challenging due to the structural complexity and the presence of the electrochemical potential. Including the potential explicitly in the quantum mechanical simulations is equivalent to simulating systems with a surface charge. For realistic relationships between the potential and the surface charge (i.e., the capacity), the solvent and counter charge need to be considered. The solvent and electrolyte description are limited by the computational power: either molecules and ions are included explicitly, but the phase-space sampling is at least 10 times too small to reach convergence or implicit solvent and electrolyte descriptions are adopted which suffer from a lack of realism. Both approaches suffer from a lack of validation against directly comparable experimental data. Furthermore, the limitations of density functional theory in terms of accuracy are critical for these metal/liquid interfaces. Nevertheless, the atomistic insight in electrocatalytic interfaces allow insights with unprecedented details. The joint theoretical and experimental efforts to design non-noble hydrogen evolution catalysts are discussed as an example for the success of theory to spur and accelerate experimental discoveries.

\section{Graphical/Visual Abstract and Caption}




\section{Poisson-Boltzmann Equation}

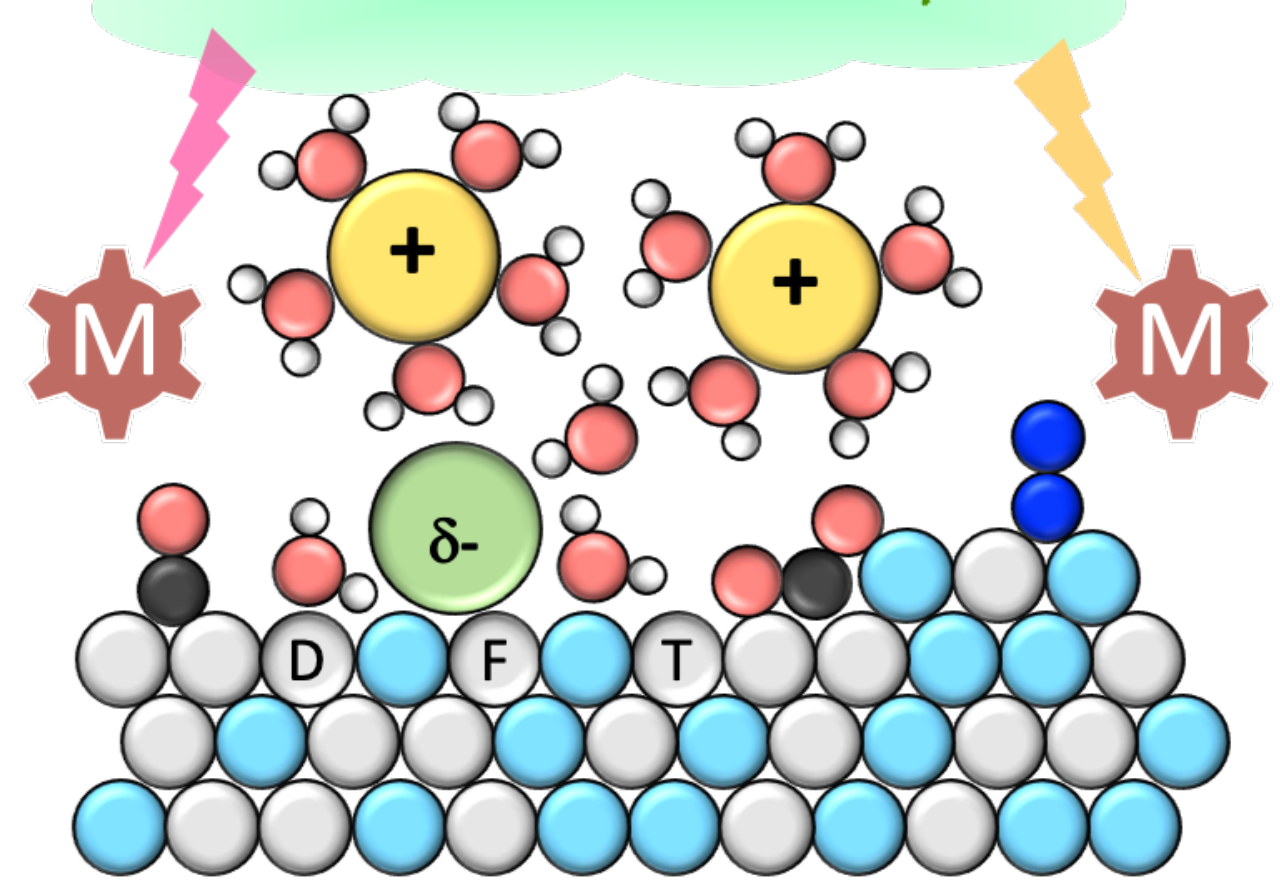

\section{INTRODUCTION}

Electrochemistry is a key technology to overcome our fossil energy based economy and energymanagement. $(1,2)$ The common characteristic of electrochemical processes is the importance of interfaces: Even for molecular electrochemistry, where the reaction is conceptually occurring in bulk solution, the need to exchange electrons with the electrode limits the reaction to the diffusion layer. Indeed, in certain cases the impact of the electric double layer on such reactions has been evidenced. (3-5) The major efforts for understanding electrified interfaces is devoted to batteries $(6,7)$ and electrocatalysis, $(8,9)$ with the more historical applications in metal-plating and corrosion(10-12) being more sparse, despite enormous economic importance (corrosion generates costs of $\sim 3 \%$ of the gross-domestic-product).(13) For batteries, the aim is to extend the cyclability of Li batteries and improve their safety via reduced dendrite formation propensity.(14) The development of $\mathrm{Na}$ or $\mathrm{Mg}$ based batteries, is also a hot topic.(15) At the intersection between batteries and electrocatalysis are the super- and pseudo-capacitors, which allow a short-term storage of electrical energy.(16) These electrochemical devices are essentially constituted of interfaces and still poorly understood due to their complex morphology.(17) Heterogeneous electrocatalysis, which is the main object of this review, combines the power of electrochemistry and heterogeneous catalysis to drive and accelerate chemical reactions, respectively. This catalytic activity is located at the electrode surface, where a (mostly liquid) electrolyte ensures the transport of ionic species.

The typical electrocatalyst is composed of a metallic surface immersed in an electrolytic solution, i.e., a solvent containing the dissolved electrolyte. However, there is a large diversity of electrocatalytic devices, ranging from gas-diffusion electrodes with only very small liquid layers at roomtemperature(18) to solid-oxide fuel cells and electrolyzers(19) which operate at $\sim 500 \mathrm{~K}$ and rely on a 
solid electrolyte. Similarly, in the electrochemical promotion of catalysis,(20) the catalyst is supported on a solid electrolyte and it is the application of the electrochemical potential that tunes the chemical reactivity. Another axis of diversity opens when considering the nature of the electrocatalyst: while transition metals are most common and Pt being the prototypical electrocatalyst, sp-metals such as Sn or Bi have also attracted attention.(21) Furthermore, non-noble transition metal oxides, sulphides, phosphides etc. have a great potential to replace the rare and expensive Pt catalysts.(22-24) More exotic electrocatalysts exploit intermetallics,(25) MXenes(26) and transition metal doped carbon nitrides. $(27,28)$ These materials generally undergo modifications upon their use as electrocatalysts and eventually they age and deactivate. This leads to challenges regarding the determination of the relevant surface state and interfaces.(29) However, since these challenges resemble very much the ones faced in heterogeneous catalysis in general, $(30,31)$ we do not treat them herein.

Today, the main application of electrocatalysis is the electrolysis of water to generate ultra-pure hydrogen and, in the reverse direction, fuel cells.(32) The use of other fuels than hydrogen (e.g., ethanol or boron hydrides) is characteristic of the research of novel electrocatalysts for oxidation reactions,(33) while electro-reduction catalysts are mostly devoted to $\mathrm{CO}_{2}(34)$ and, increasingly, $\mathrm{N}_{2}$ reduction.(35) Application of electrocatalysis for electrosynthesis of organic molecules is comparably rare.(36)

The large diversity of electrified interfaces in general and even when focusing on heterogeneous electrocatalysis constitutes the first major challenge for their computational modelling: Even with today computational resources, a single methodology is unlikely to be powerful enough to reliably describe the disorderd solid-electrolyte interphases found in batteries, the impact of morphology on super-capacitor performance and bond-breaking and formation processes at the active sites on electrocatalysts. Hence, the relevant scale (from atoms to centimeters) dictates the use of given modelling methods or imposes the use of mulit-scale models.

Even though electrocatalysis is intrinsically a multiscale process, with charge- and mass-transport being of significant technological importance,(37) we herein focus on the atomic scale: The atomic scale is where the intrinsic limitations of the catalysts are found and where the methods that allow to understand electrocatalysis can be almost directly transposed to other electrified interfaces. We refer to the appropriate reviews for the larger scale models (applied to fuel cells) and do not treat them herein.(38-40)

The remainder of this review is structured into five sections. The short first one discusses conceptual questions that haunt the atomistic modelling of electrocatalysis. The second section gives an extensive overview on techniques to model the electrified interface. The third section discusses outstanding challenges that wait for solutions and the fourth section presents the significant insights obtained by atomistic modelling of electrocatalysis for the hydrogen evolution reaction. The fifth section briefly discusses possibilities to more thoroughly validate the atomistic models.

\section{VOLCANO CURVES, OVERPOTENTIAL AND THEIR POTENTIAL DEPENDENCE}

Computational modelling complements experimental observations and is, in principle, able to suggest the most promising combinations of electrode material, electrolyte and electrochemical potential. However, given that the methodology required for these simulations is still in its infancy, it cannot come as a surprise that there is no consensus in the community and harsh criticism regarding the 
usefulness of computational modelling to complement experimental studies is partially justified. $(41,42)$ Here, we first introduce the notion of the volcano diagrams and their limitations and then tackle the overpotential and how to connect the experimental overpotential to the quantities from atomistic modelling.

Sabatier's principle of heterogeneous catalysis states that the optimal catalyst binds adsorbates and intermediates with an appropriate binding energy: if intermediates are bound too strongly, they block active sites and if they are too weakly adsorbed, they are not sufficiently present on the surface to allow for high reaction rates.(43) This principle still holds in electrocatalysis: indeed, it is almost trivial that intermediates need to be stabilized appropriately in order for the reaction to proceed without major kinetic limitations. In practice, for a family of catalysts, the Sabatier principle is most often represented as a two-dimensional graph, with a descriptor (possibly the binding energy of the limiting intermediate) serving as the $x$-axis and the $y$-axis representing the activity. These graphs are commonly called "volcano plot", as they tend to show a unique maximum activity. Following an earlier proposal by Parsons, (44) Trasatti was the first to publish an electrocatalytic volcano curve, applied to the case of the hydrogen evolution reaction (HER).(45) While in principle any descriptor that leads to a exploitable volcano curve works, the determination of this descriptor ( $x$-axis) is not always possible experimentally. Therefore, most of the volcano curves in the literature rely on Density Functional Theory (DFT) computations(42) as pioneered by Nørskov and co-workers.(46) Nevertheless, as we will discuss in section 3, the binding energy of intermediates depend, in principle, on the full electrified electrode/electrolyte interface and even on the target potential. It turns out that in practice simple descriptors, such as the adsorption energy of atoms (typically $\mathrm{H}, \mathrm{O}, \mathrm{N}$ and $\mathrm{C}$ ) are a close enough proxy for the determination of volcano curves. The advantage of these very simple descriptors is that they allow to screen a very large number of similar materials (e.g., surface alloys) at modest computational cost. $(47,48,8,9,49)$

The slopes of the volcano tend to be linear. This has led to a second highly important concept: the "universal" scaling relationships,(50-52) which describe the fact that the adsorption energy of various intermediates cannot be tuned independently. Overcoming these scaling relations has, therefore, been an important goal in the recent years, with the main approaches being (i) bifunctionality, e.g., alloys (ii) addition of promoters, (iii) functionalization of the surface via ligands (iv) electrolyte and solvent engineering, (v) interfacial sites, i.e., heterofunctional catalysts and (vi) confinement (see Fig.

Figure 1).(53-55) 


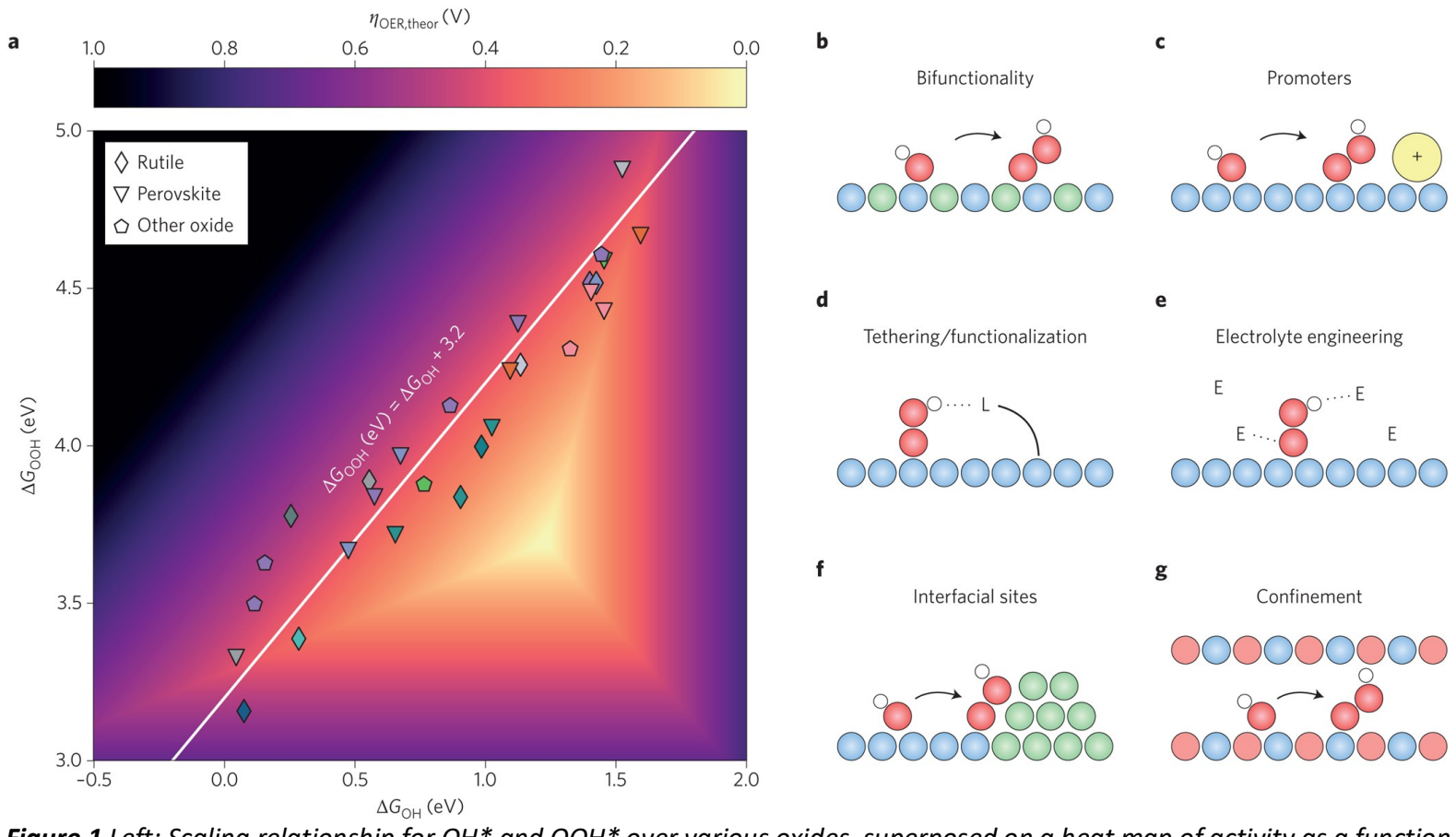

Figure 1 Left: Scaling relationship for $\mathrm{OH}^{*}$ and $\mathrm{OOH}^{*}$ over various oxides, superposed on a heat map of activity as a function of these two variables. Note that the optimum (brightest color) is does not lie on the scaling line (a). Right: Examples of how to overcome the scaling relation by introducing adsorbate specific stabilizations. Bifunctionality (b) on alloys or mixed metal/oxide/sulfide catalysts, promoters in terms of specifically adsorbed ions (c), ligands (d) or electrolyte molecules (e) can stabilize various adsorbates specifically. Three dimensional active sites such as interfaces between two materials (f) or confinement $(\boldsymbol{g})$ can also provide opportunities to break scaling relationships. Differently colored circles indicate distinct elements. Reproduced from(54), copyright Nature Publishing Group.

Closely related to the volcano curves in electrocatalysis is the concept of overpotential and the exchange current density: In short, the overpotential $(\eta)$ is the minimal voltage that is necessary to significantly accelerate an electrochemical reaction, while the exchange-current density $\left(i_{0}\right)$ is the current density at the equilibrium potential $U^{\circ}$, i.e. zero overpotential. The standard equilibrium potential is connected to the free reaction energy $\Delta \mathrm{G}^{\circ}$ via:

$$
\Delta G^{\circ}=-n F U^{\circ}
$$

Where $\mathrm{n}$ is the number of electrons exchanged and F Faraday's constant. The more general form of this equation is known as the Nernst equation. For a half-cell reduction reaction it reads:

$$
U_{\text {red }}=U_{\text {red }}^{\circ}-\frac{R T}{n F} \ln \frac{a_{r e d}}{a_{o x}}
$$

Where $\mathrm{R}$ is the universal gas constant, $\mathrm{T}$ the temperature and $\mathrm{a}_{\mathrm{red}}$ and $\mathrm{a}_{\mathrm{ox}}$ the activities of the reduced and oxidized species, respectively. Eq. 1 and Eq. 2 together determine the minimum cell potential that is required for the reaction to be spontaneous, i.e., that the reaction free energy is zero or negative. If a larger potential difference is applied, the reaction is driven by the external power source. In the reverse direction, the overpotential measures the minimal energy loss of a battery or fuel cell.

In the simplest systems, the Tafel equation provides the link between overpotential and the exchangecurrent density:

$$
\eta=A \cdot \log _{10}\left(\frac{i}{i_{0}}\right)
$$

Where $A$ is the so called Tafel slope and $i$ the current density. Figure $\mathbf{2}$ shows a model Tafel plot to illustrate the connection between the Tafel slope and the exchange current density. It also becomes 
evident that the Tafel plot relies on a linear approximation in order to extract information out of the measured current densities.

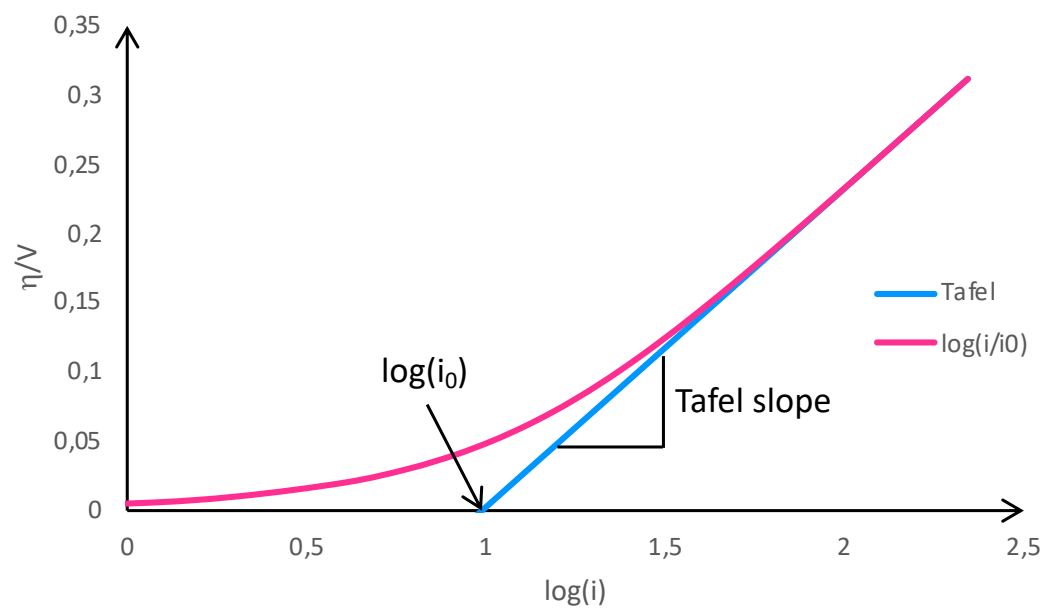

Figure 2 Schematic plot of the overpotential ( $\eta$ ) vs the logarithm of the current density (pink). The Tafel slope and the exchange current density $i_{0}$ as determined by the linear fit (Tafel approximation) are indicated (blue).

Tafel slopes depend on the reaction mechanism, i.e., if the electrochemical step (i.e., an elementary steps where electrons are exchanged with the electrode) is rate determinating or not and if it is preceded by chemical or electrochemical steps, as nicely summarized in ref (56). Here, chemical steps refer to elementary steps that preserve the formal number of electroactive species in the system. Typical values for Tafel slopes at room temperature vary between 30-120 mV/decade. Detailed microkinetic models show that the connection between the observed Tafel slope and the reaction mechanism is far from straightforward.(57-59) For atomistic modelling of electrocatalysis, the limitation is almost immediately clear: The overpotential as defined in Eq. $\mathbf{3}$ is intrinsically a kinetic quantity and thus depends on the larger scales. The crudest approximations for obtaining these kinetic characteristics is the framework of microkinetics, where mean-field differential equations are solved.(60) Lattice based kinetic Monte Carlo simulations, where the coverages of intermediates and their spatial arrangements are explicitly simulated, are more realistic, but also computationally much more involved.(61,62) A variety of other multi-scale and multi-physics simulations have been developed in order to include the macroscopic properties such as the charge and mass-transfer, viscosity and so on. (38-40) Since performing these simulations exclusively from first principles information is unfeasible in practice, a number of approximations have been adopted. While some studies prefer to approximate prefactors in the mean-field differential equations which then are solved under steady-state conditions, the most common approximation is the most drastic one: neglecting all detailed kinetics and focusing exclusively on the thermodynamics of the reaction pathway. This leads to the concept of "thermodynamic overpotential" $\left(\eta_{\mathrm{TD}}\right)$. $\eta_{\mathrm{TD}}$ is the potential which is necessary to make all the electrochemical steps either athermic or exothermic. $(60,63)$ Assuming that the reaction mechanism only involves electrochemical steps and no chemical steps, $\eta_{\text {TD }}$ ensures that there are no thermodynamic barriers anymore. In contrast with the kinetic modelling, determining $\eta_{\text {TD }}$ is well defined at an atomistic level. Furthermore, the influence of the level of theory (inclusion of the surface polarization, coverage and electrolyte effects and so on) can be assessed with greater ease than for a full kinetic study. However, this approximation is very crude: in particular, the reaction barriers and the thermodynamics of chemical steps, as opposed to electrochemical steps, are 
difficult to include within this framework. Hence, while $\eta_{\text {TD }}$ tends to be a relevant quantity for screening catalysts, it is clearly not enough for a detailed understanding of reaction mechanism.(64) The second key issue with $\eta_{\text {TD }}$ is that it is such a crude approximation that it is not even clear what experimental quantity it should be compared or correlated to: Is it the overpotential based on the Tafel relation, i.e., when the linear approximation becomes valid? - The overpotential as determined experimentally based on a limiting current density, often taken as $10 \mathrm{~mA} / \mathrm{cm}^{2}$ and thus more commonly available than a complete Tafel analysis? - Or correlated to the exchange current density, which is most closely related to thermodynamic barriers?

The discussion of $\eta_{T D}$ is closely related to the volcano curves, as it is one of the most frequently used theoretical measures for the activity of a given family of catalysts. To simplify the determination of theoretical volcano plots even further, the binding energy of intermediates in the absence of an applied potential can be used. This, however, is an even more dramatic approximation, as it assumes that the binding energy of all intermediates behave the same as a function of the potential. This later approximation is equivalent to assume that the Tafel slope does not change as a function of the potential, an assumption that is rarely fulfilled for effective electrocatalysts.(65-67)

\section{APPROACHES TO MODEL THE ELECTRIFIED INTERFACE}

\subsection{Early days}

In this section, we adopt a historical and largely chronological point of view. As very nicely described by Bockris in 1971, the existence of overpotentials, which is a key concept in electrocatalysis, was still very ill understood and even underrated by the community for many decades.(68) Therefore, it cannot come as a surprise that atomistic modelling via quantum chemistry was very slow to shed light on this tantalizing problem. Nevertheless, the group of Anderson pioneered the use of quantum-chemical computations to investigate electrocatalysis already in the late 70ies.(69-71) Theses early (semiempirical) approaches already tried to take the electrochemical potential into account, for instance by shifting the ionization energy of metal atoms. This approach could even be validated against experimental changes in infra-red frequencies as a function of the electrode potential.(70) Similarly and only slightly later, the "dipped adcluster" model of Nakatsuij proposed to perform Hartree-Fock computations with an applied potential based on a cluster model.(72) However, the electronic structure of the cluster features a HOMO-LUMO gap in contrast with metals that have a continuous density of state around the Fermi level. This gap turned out to interfere strongly, so that the physics of a metallic electrode(73) could not be retrieved. As most first-principles studies, the golden days only started in the late 1990s. The work by Bureau and Lecayon introduced the use of grand-canonical DFT (GC-DFT) for simulating electrocatalysis in 1997.(74) To make it simple (for details see section 3.3.1), this approach consists in varying the number of electrons. Thus, the Fermi-level, i.e., the workfunction and thus the electrochemical potential is tuned. Even though the actual model was still based on a cluster and the treatment of the solvent and countercharge was not addressed, the theoretical framework and its connection with conceptual DFT, such as the importance of the Fukui function, was fully laid out. Around the same time, Anderson's group introduced a second key concept in the atomistic modelling of electrocatalysis, which is the thermodynamic cycle that allows to link a given computation to the electrochemical potential. (75) The approach to deal with electrochemical steps closely follows the early arguments of Bockris(76) which avoids the need to know the energy of the (solvated) electron independently. Although performing computations in the gas-phase, Anderson 
already assessed the potential dependence not only of electrochemical steps, but also for reaction barriers.(77) Coming from the molecular chemistry community, these pioneering computations basically disregarded the explicit influence of the electrode or approximated the electrode by single atoms. (78) Bredas and co-woerks validated he use of grand-canonical DFT by determining Stark tuning slopes, for which a model charge-distribution was used to stabilize the surface charges.(79) Alternatively, explicit countercharges were used.(80) To simplify such computations that probe the effect of the local electric field, the use of a uniform electric field was proposed.(81) However, the relation between the field-dependent results and the actual electrochemical potential is not immediate and this approach was thus not very successful for electrocatalysis. (82) Along the lines of grand-canonical DFT, the group of Alavi and Sprik turned to the problem of using periodic computations for treating solution redox chemistry.(83) The periodicity introduces the delicate issue of how to neutralize the unit cell in order to avoid a diverging Coulomb potential of periodic charges. In this pioneering work, the authors relied on a homogeneous background charge without discussing the issue in details. The use of the homogeneous background charge was subsequently criticized since it introduces artificial energy terms and forces.(84) Nevertheless, even in this study the conclusion was that for some applications it remains the only practical solution, although the introduced errors are difficult to assess. Alavi and co-workers later provided examples for the use of a Gaussian countercharge sheet in periodic simulations instead of the homogeneous background charge. However, the scheme was still applied in vacuum.(85) For determining redox-potentials in solution, Sprik and co-workers heavily rely on the homogeneous background charge, last but not least since no clear, practical alternative exists for small, periodic simulation cells. $(86,87)$ Turning back to heterogeneous electrocatalysis, it was again Anderson who, after summarizing his "reaction center" cluster approach $(88)$ turned to the use of periodic slabs. $(89,90)$ Compared to the now so popular method called computational hydrogen electrode (CHE), which we will discuss in the next subsection, Andersons scheme is virtually identical. The only substantial difference is that he did not advocate the use of the very convenient $\mathrm{H}_{2}$ molecule as a reference compound but rather the $\mathrm{OH}$ radical. This scientifically small detail seems to have tipped the balance, so that today the concept of CHE and the surge of computations for heterogeneous catalysis is ascribed to the Norskov group instead of Anderson, who, at the very same time, had already two decades of experience with the atomistic modelling of electrocatalysis.

\subsection{The CHE revolution}

The field of atomistic modeling of electrocatalysis has made an enormous leap forward with the introduction of the computational hydrogen electrode (CHE) by Norskov et al.(60) in 2004. The CHE method enables the evaluation of the relative free energies of intermediates along an electrochemical reaction network. Each elementary step is formulated as a removal or addition of the electroactive species, $\left(\mathrm{H}^{+}+\mathrm{e}^{-}\right)$. The pathway of water oxidation, relevant to water electrolysis, provides a typical example:

$$
\begin{aligned}
\text { 1: } \mathrm{H}_{2} \mathrm{O}+* & \rightarrow \mathrm{OH}^{*}+\mathrm{H}^{+}+\mathrm{e}^{-} \\
\text {2: } \mathrm{OH}^{*} & \rightarrow \mathrm{O}^{*}+\mathrm{H}^{+}+\mathrm{e}^{-} \\
\text {3: } \mathrm{H}_{2} \mathrm{O}+\mathrm{O}^{*} & \rightarrow \mathrm{OOH}^{*}+\mathrm{H}^{+}+\mathrm{e}^{-} \\
\text {4: } \mathrm{OOH}^{*} & \rightarrow \mathrm{O}_{2}+*+\mathrm{H}^{+}+\mathrm{e}^{-}
\end{aligned}
$$

The adsorbates are indicated via an asterisk and a single asterisk * denotes an empty reactive site on the catalyst. At each (electrochemical) step, $\left(\mathrm{H}^{+}+\mathrm{e}^{-}\right)$are abstracted from the adsorbates. The free energy $\Delta \mathrm{G}$ for each step in the oxidation reaction can then be expressed as below: 


$$
\begin{gathered}
\Delta \mathrm{G}_{1}=\mathrm{G}_{\mathrm{OH} *}-\mathrm{G}_{\mathrm{H} 2 \mathrm{O}}-\mathrm{G}^{*}+\mu_{\mathrm{H}+}+\mu_{\mathrm{e}-} \\
\Delta \mathrm{G}_{2}=\mathrm{G}_{\mathrm{O} *}-\mathrm{G}_{\mathrm{OH} *}+\mu_{\mathrm{H}+}+\mu_{\mathrm{e}-} \\
\Delta \mathrm{G}_{3}=\mathrm{G}_{\mathrm{OOH} *}-\mathrm{G}_{\mathrm{OOH} *}-\mathrm{G}_{\mathrm{H} 2 \mathrm{O}}+\mu_{\mathrm{H}+}+\mu_{\mathrm{e}-} . \\
\Delta \mathrm{G}_{4}=\mathrm{G}_{\mathrm{O} 2}+\mathrm{G}^{*}-\mathrm{G}_{\mathrm{OOH} *}+\mu_{\mathrm{H}+}+\mu_{\mathrm{e}-}
\end{gathered}
$$

$G_{x}$ is the free energy of each specie involved and it can be determined by DFT computations. It typically contains the electronic energy $E$, the entropy $S$, and a zero-point energy correction $Z P E: G=E+Z P E$ $-\mathrm{TS} . \mu_{\mathrm{H}+}$ and $\mu_{\mathrm{e}-}$ are the electrochemical potentials of the removed protons and electrons, respectively, which are not directly determined by ab initio computations. Instead, the beautifully simple method of Norskov is based on the observation that under standard conditions ( $\mathrm{pH}=0, \mathrm{~T}=298 \mathrm{~K}, \mathrm{p}=1$ bar), defining $0 \mathrm{~V}$ of the standard hydrogen electrode $(\mathrm{SHE})$, there is an equilibrium $(\Delta \mathrm{G}=0)$ between hydrogen molecules in the gas phase and solvated protons and electrons:

$$
1 / 2 \mathrm{H}_{2(\mathrm{~g})} \leftrightarrow \mathrm{H}^{+}+\mathrm{e}^{-}
$$

Hence, the free energy of the $\mathrm{H}_{2}$ molecule in the gas phase, which is trivial to compute, can be used to determine the combined energy of $(\mathrm{H}++\mathrm{e}-)$ :

$$
\mu_{\mathrm{H}+}(\mathrm{U}=0 \text { vs } \mathrm{SHE})+\mu_{\mathrm{e}-}(\mathrm{U}=0 \text { vs } \mathrm{SHE})=1 / 2 \mathrm{G}_{\mathrm{H} 2}
$$

which is much easier than computing the solvation energy of the protons and electrons. When changing the potential away from $0 \mathrm{~V}$ vs SHE a term neU has to be added where $\mathrm{n}$ is the number of exchange electrons, e the elementary charge and $U$ the potential, all in suitable units. This term is then added to each electrochemical step. Together with a free energy correction for the proton activity that depends on the $\mathrm{pH}$ of solution, the free energy of an electrochemical oxidation step according to $\mathrm{CHE}$ is written as:

$$
\Delta G_{x}(U)=\Delta G_{x}-n e U+k_{B} T p H \ln (10)
$$

Use of Eq. 8 naturally shifts the focus of $\mathrm{CHE}$ from SHE to the reversible hydrogen electrode (RHE), i.e., to a $\mathrm{pH}$ independent reference electrode. Indeed, $\mathrm{CHE}$ is not well adapted to study (explicit) pH effects and implicitly assumes that the neutral surface state computed by DFT is representative of $0 \mathrm{~V}$ vs RHE.

In order for the overall reaction to occur without thermodynamic barriers, $\Delta G_{x}(U)$ has to be either zero or negative. The computation of free energy differences along a pathway allows to estimate this potential $U_{\text {min, }}$ which, when compared to the equilibrium potential $U^{\circ}$ defines the thermodynamic overpotential $\eta_{\mathrm{TD}}$, itself by definition always positive. The great simplicity of CHE relies on a second approximation: the solvation effects are assumed to be negligible, which means that only computations in the gas-phase are necessary to evaluate electrocatalytic reaction pathways. However, this second assumption is not necessary and some authors use the CHE in conjunction with solvation effects. $(91,92)$

A lot of DFT based research in electrocatalysis studies relies on the CHE approach as a tool to design the catalytic activities of different materials $(47,49,93,94)$, determine phase diagrams $(95)$ and estimate the free energy of various electroreduction and electrooxidation reactions such as the oxygen reduction reaction,(96) the oxygen evolution reaction,(97) the hydrogen evolution reaction (HER)(98$100)$, the $\mathrm{CO}_{2}$ reduction reaction(101-103), and the $\mathrm{N}_{2}$ reduction reaction(104,105).

Despite these exploitable results, CHE has several limitations and unanswered questions remain related to the approximations in establishing the model. In the original applications of the $\mathrm{CHE}$, solvation effects were assumed to play a minor role for the energetics of neutral reaction intermediates and thus omitted. The interaction between electrode, adsorbates and the solvent is neglected. In addition, the electrode potential in this method is not an explicit variable in the quantum mechanical calculations. The electronic energies are only measured for electroneutral entities independently from the electrochemical potential as the electron transfer reaction is always coupled. This is problematic in view of the accumulating experimental evidence that decoupled pathways are 
also key.(106-109) All these interrelated limitations of the CHE method are addressed by the "beyond the CHE" methods discussed in the next subsections, which explicitly model the electrochemical potential and the electrode/electrolyte interface.

To summarize, the CHE approach often provides information on adsorption trends but the role of the chemical electrolyte/electrode interface is outside the scope of this type of model. It only allows the evaluation of the thermodynamics of the elementary reaction steps. Generally, activation barriers for charge transfer reactions are ignored, even though some schemes have been developed to approximate the potential dependence of barriers in a CHE-like spirit. (110-112)

In the next section, we will review the methods to model the electrochemical with increased realism compared to $\mathrm{CHE}$, which allow to understand the interaction between adsorbates and the electrified, solvated electrode. These methods are based on explicitly changing the surface charge density of the electrode surface, so that activation energies and chemical steps, can also be assessed at a given potential.

\subsection{Beyond the computational hydrogen electrode}

Any method that aims to go beyond the first order $\mathrm{CHE}$ approximation should describe the interactions between the adsorbates and the electrified electrode. This can be either achieved via potential dependent density functional theory computations (section 3.3.1) or model Hamiltonians (section 3.3.2). We here emphasize that to be considered an improvement beyond the $\mathrm{CHE}$, the methods have to include the energetics as expressed by Eq. 7 and 8 . Figure 3 summarizes the various possibilities to treat the electrochemical interface, ranging from the vacuum interface at zero charge to grandcanonical DFT coupled to molecular dynamics. The aim of the figure is to illustrate the panel of available models and that the timeline is scattered across the two-dimensional surface.

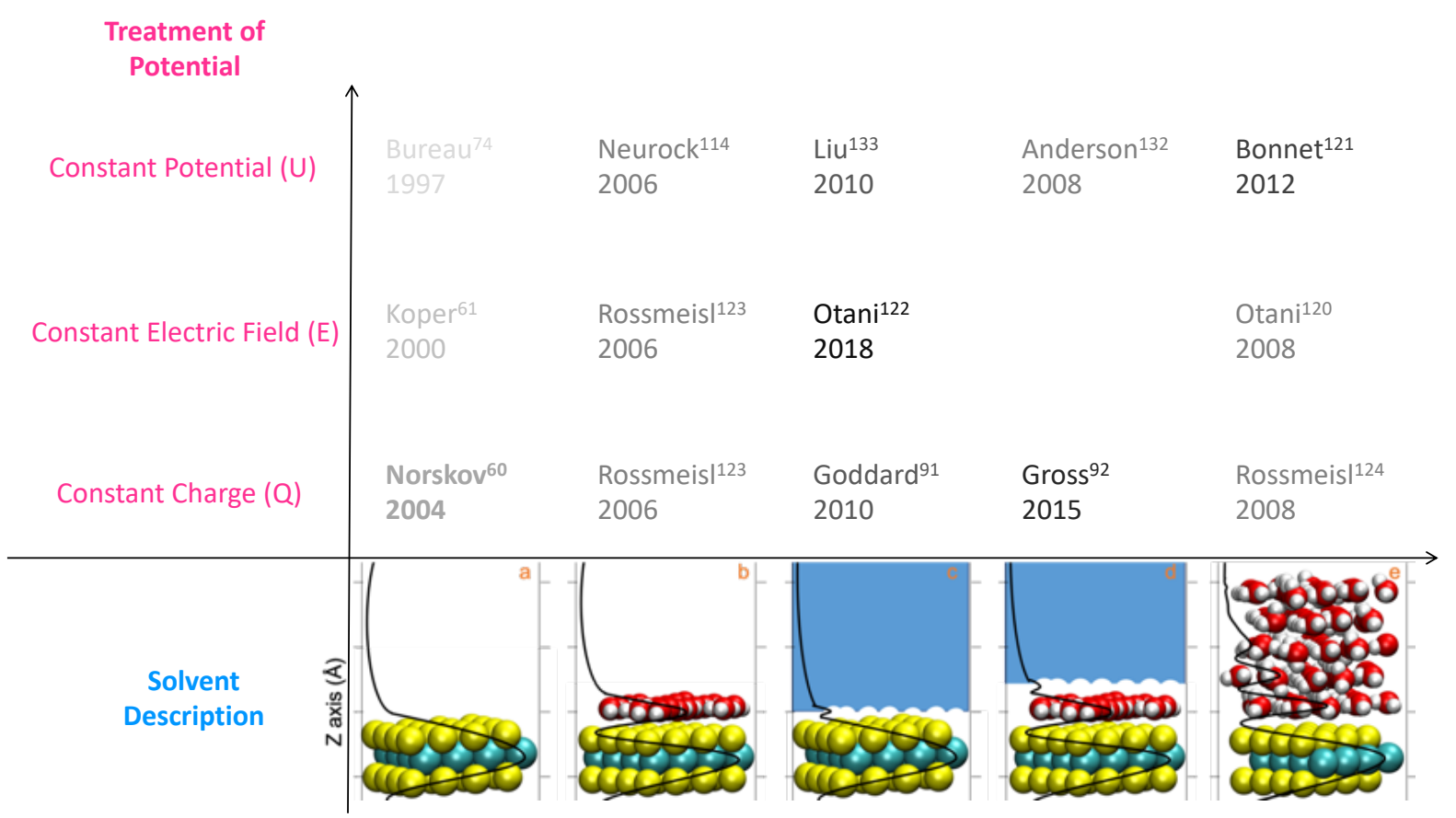

Figure 3 Representation of the key contributions introducing various descriptions of the electrocatalytic interface. The superscript refers to the reference to the corresponding article, while the year is given below the lead author name. The grey scale highlights the chronological ordering. Note, that other groups have applied these techniques in various contexts and 
some of the methods have been developed several times independently with slight variations, but we have tried to highlight the earliest contributions. The horizontal axis is indicative of the treatment of the solvent, with the black line symbolizing the electrostatic potential. (a) corresponds to vacuum, (b) the combination of a small number of solvent molecules at the vacuum interface, (c) the use of an implicit solvent, (d) the combination of implicit solvent (light blue) and explicit solvent molecules and (e) the use of a fully explicit description of the liquid phase. The CHE cornerstone is highlighted in bold. The vertical axis represents the description of the electrochemical potential. Constant charge approaches typically work with neutral surfaces. Constant electric fields can be used as a proxy for the electrochemical potential under certain assumptions (typically the thickness of the double layer). Truly constant potential approaches are typically relying on grand-canonical DFT.

\subsubsection{Grand-Canonical Density Functional Theory}

As discussed in section 3.1, grand canonical DFT is the framework which allows to perform electronic structure computations at a given electrochemical potential to mimic the experimental conditions. Hence, the electronic grand-canonical (free) energy $F$ now depends on the electrochemical potential $U$, instead of being evaluated at zero charge as in the CHE approximation:

$$
F(U)=E(U)-q(U) * U \approx E\left(U_{0}\right)-\frac{1}{2} C\left(U-U_{0}\right)^{2}
$$

Where $E(U)$ is the electronic energy at potential $U$, the (surface) charge $q_{\text {surf }}$ is positive if electrons are removed and negative when electrons are added. $U_{0}$ is the workfunction of the system at zero charge and thus $E\left(U_{0}\right)$ is the energy which would be used in the CHE framework. The second equality is valid for metallic (gap-less) systems, for which the grand-canonical energy $F(U)$ can be expanded to second order in terms of the capacitance $\mathrm{C}$ of the system. In contrast to $\mathrm{CHE}$, the explicit dependence of the electronic energies on the electrochemical potential capture the potential-dependence of formally chemical steps, $(113,64)$ e.g. the desorption of $\mathrm{O}_{2}$ depicted in Figure 4 . The last ten years or so have seen several computational approaches that all rely on Eq. 9 to compute potential dependent adsorption energies on periodic surfaces. There are two main characteristics that distinguish them: how the surface charge is neutralized and how the solvent is described.

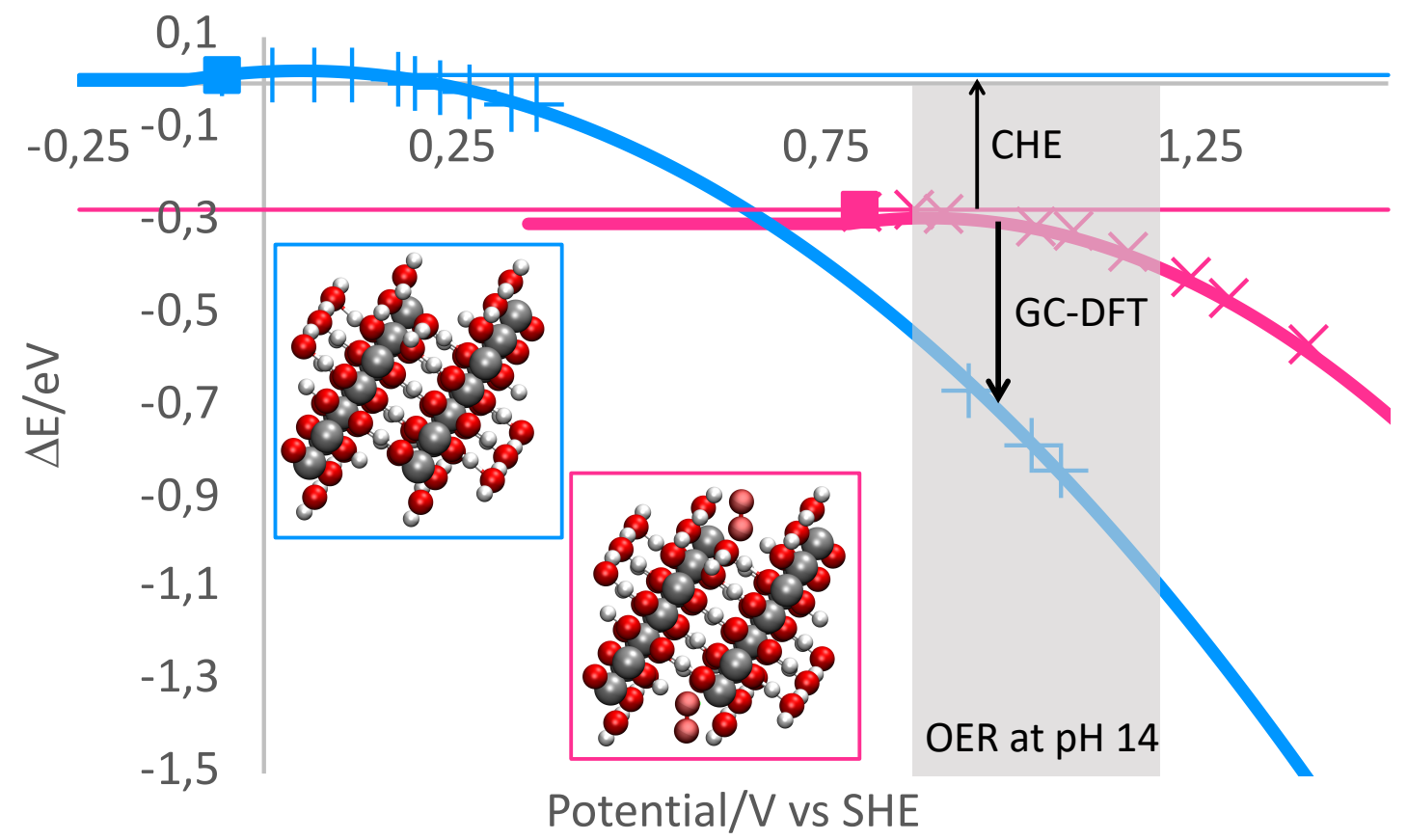

Figure 4 Comparison of CHE (horizontal lines and solid points) and grand-canonical DFT (empty symbols and thick lines) and grand-canonical DFT (GC-DFT) results for the energy difference of two intermediates during the oxygen evolution reaction (OER) over $\mathrm{COOOH}$ (a semi-conductor) in basic conditions. The intermediate in pink (crosses) has $\mathrm{O}_{2}$ bound to the catalyst, while $\mathrm{O}_{2}$ is desorbed in the intermediate in blue (plus signs). The desorbing $\mathrm{O}_{2}$ molecule is highlighted in pale red in the 
structural representation, which also exemplifies the use of symmetric unit cells to avoid surface dipole moments and obtain unambigious workfunctions. The data is taken from ref. (64)

Fundamentally, a charged periodic system has a divergent Coulomb energy. Hence, the periodic unit cell has to be neutralized. However, the countercharge can take various forms. Neurock and coworkers were among the first to apply grand-canonical DFT to electrocatalysis on a routine basis for electrocatalysis.(114-116) In their scheme, a homogenous background charge is used to neutralized the simulation cell. However, since part of the system is metallic, a simple energy correction with respect to the neutral system allows to obtain physically relevant electronic energies. In practice, they used static water (ice) layers to simulate the solvent, even though later the approach has been transposed to the use of implicit solvation models. $(117,118)$ This model does not require any modification of the computational software, explaining its attractiveness from a user perspective. Performing different computations with varying numbers of electrons, a quadratic fit (right hand side of Eq. 9) allows to determine the energies as functions of the electrochemical potential at moderate computational cost. Finally, the equilibrium (redox) potential is calculated as an intersecting point between two curves.

Almost at the same time, Otani and Sugino(119-122) presented another model which avoids the use of the homogeneous background charge. This approach is called "effective screening medium" (ESM) and exploits Green's function techniques to formally treat a non-repeated slab along the direction of the surface normal. The Green's function allows to impose two different electron potentials across the unit cell, so that the "bottom" can be imposed to have the metal bulk potential, while the potential away from the surface mimics the counter-electrode. The model is most suitable to simulate situations with a linear electric field in the solvent region and requires extensive modifications of the electronic structure code, so that it is not widely used. Like for other approaches that explicitly rely on an electric field,(123) the assumptions about the thickness of the double layer introduces uncertainties which are require somewhat arbitrary decisions.

The homogeneous background charge,(114) ESM(119) or the Gaussian charge sheets(85) can only qualitatively represent the actual electric double layer and introduce, therefore, ad hoc assumptions. To avoid using these artificial treatments for countercharges, Rossmeisl and co-workers(124-127) proposed to control the electrode potential by changing the number of hydrogen atoms that dissociate into protons and electrons so that the total charge remains neutral. However, adding or removing one electron and one proton to or from the practical (i.e., relatively small) unit cells causes a drastic change in the calculated electrode potential, since fractional protons(128) are not considered as an option. Consequently, controlling the electrode potential within a desired narrow range is difficult. Hence, the reaction energies for electrochemical steps (e.g., adsorption of a proton) need to extrapolated to infinite supercell sizes. Furthermore, just like for the ice-layers used by Neurock,(114) the description of solvation effects via static solvent layers is very approximate, but highly important for these charged species. Note, that these arguments "against" the generalized CHE method of Rossmeisl and co-workers are only of practical nature. In principle, the generalized CHE method with varying numbers of explicit cations/anions is sound and if sufficiently large unit cells could be afforded, while achieving a representative sampling of the solvent (and electrolyte) phase-space, it would lead to a realistic description of the electrified interface (up to the errors of DFT itself, discussed in section 4.2). However, we need to keep in mind that such simulations imply a grand-canonical treatment of 
the electrolyte and/or electroactive species. Based on the experience with constant $\mathrm{pH}$ simulations at the classical force field level(129,130), which remain a field of active research, and the practical and conceptual issues discussed in section 4.1, we expect that these simulations remain rather impractical for routine applications at least for another ten years or so. Nevertheless, promising results have been obtained for the simulation of cyclic voltammograms.(131)

An elegant solution is to modify the surface charge and describe the double layer according the solution of the (modified) Poisson-Boltzmann (mPB) equation.(132-138) The Poisson-Boltzmann equation provides a mean-field description, obtained by placing the countercharge in the solvent region according to the local effective electrostatic potential. Compared to the homogeneous background charge or the Gaussian charge-sheet, the resulting ion distributions are much more realistic. The formal theory behind this combination has also been called joint DFT (JDFT) by Arias and co-workers, $(134,139)$ making reference to the fact that these ion- and solvent distributions can be formally generated by classical, "molecular" density functional theory, while the electronic structure of the slab is determined by the more conventional quantum mechanical, "electronic" DFT. The mPB equations do not automatically satisfy the charge neutrality constraint, which necessitates further corrections. $(140,141)$ Thus, even the electronic DFT combined with mPB models need in practice either to use Lagrange multipliers in order to enforce the charge neutrality,(140) to employ simple background charges(142) or to utilize modified mPB boundary conditions(119) which mimic neutralization by image charges to treat the charged semi-periodic systems. This contrasts with the most popular linearized Poisson-Boltzmann equation, which automatically generates neutral unit cells. Since the linearized PB equation assumes that the countercharge is made of point charges, it neglects, however, all finite size effects, resulting in countercharge distributions that approaches the solute too closely.(143) Compared to the homogeneous background method, where an energy correction is needed that assumes that the slab is metallic, the (linearized) Poisson-Boltzmann equation is also readily compatible with semi-conductors (see Figure 4)(64) and layered electrocatalysts, $(144,145)$ making this approach universally applicable. Indeed, the advantage of the combination of DFT with the linearized Poission-Boltzmann equation is that it can be applied to any charged system, while producing physically meaningful electronic energies. Hence, we have also applied it to investigate the origin of electrochemicall promotion of catalysis (EPOC) phenomenon $(146,147)$ and to study the effect and the role of adding a base in heterogeneous catalysis (148).

\subsubsection{Model Hamiltonians}

While DFT is the most popular approach used to simulate the electrochemical reactions, it has several limits. As mentioned in section 3.1, several theories for electrochemical reactions already existed a long time before the advent of DFT, taking their origin in the theories of Marcus(149) and Hush(150) and its extended versions.(151) These treatments rely on a highly simplified representation of the valence orbital of the reactant, the electrons on the metal, their electronic interaction, and the coupling of the electron exchange to the solvent. The electrochemical potential is taken into account by shifting the reactant's valence orbital with respect to the metal. The current use of model Hamiltonians in electrocatalysis is mainly the result of Schmickler and co-workers, (152-154) who have combined Marcus theory and the Anderson-Newns model, $(155,156)$ within a tight-binding or Hückel approximation and exploiting Green's function methods. This model Hamiltonian allows to account for bond-breaking and formation and leads to a thorough understanding of trends in electrocatalysis, 
e.g. for the hydrogen evolution reaction.(157) A similar decoupling between electron transfer and the evolution of the nuclear coordinates can, in principle, be achieved via the block-localized wave function techniques(158) at the DFT level. On the one hand, the limitations of the model Hamiltonians are, of course, that they have to be set up for each system specifically, with certain terms nowadays being determined by DFT computations.(159) On the other hand, the major strength of the model Hamiltonians is the deep insight they provide and the ability to compute free-energy surfaces for electrochemical reactions as a function of the reactant's position and the solvent coordinate, a topic that we will discuss again in the next section.

\section{SELECTED CHALLENGES WHEN MODELLING ELECTROCATALYSIS}

\subsection{The rise and fall of implicit solvents}

Electrocatalysis occurs at the electrode-electrolyte interface and a very important part of the electrochemical system is the solvent and thus the associated solvation free energies.(160) Solvation free energies are statistically averaged interactions between solutes and solutions. Most of the schemes discussed in section 3 include either just a few solvent molecules(110) or a couple ice layers(161) but without performing any statistical average or, in the other extreme, they include only the statistical average, directly obtained from integral or differential equations. These two options define the typical "explicit" and "implicit" solvation models, although approaches that combine characteristic of the two are common.(162,163)

The downsides of explicit solvation models are the limited sampling of static structures, the difficulty of reaching a global energy minimum and the challenge to maintain a consistent solvation sphere for various adsorbates along a reaction pathway. Ab initio molecular dynamics (AIMD) $(126,164)$ can alleviate these drawbacks, as it allows to sample a range of solvation structures at the electrode/electrolyte interface. However, AIMD does not reach the time and length scales necessary to assure a representative sampling of the structural ensemble. Indeed, given the size of the solid/liquid interface, brute force AIMD studies have only been applied to open-circuit model systems,(165-168) or small systems, where lateral ordering is imposed. Even for these rare casestudies, the accessible $<50$ ps of dynamics are an order of magnitude smaller than what is necessary to equilibrate a pure $\mathrm{H}_{2} \mathrm{O} / \mathrm{Pt}(111)$ interface, even in the absence of ions and adsorbates.(169) To circumvent this issue, the group of Goddard has been relying extensively on pre-equilibration at a reactive force field level of theory $(170)$ before turning to the actual AIMD simulations. $(168,171,172)$ This is only possible if a suitable empirical force fields is available, which is currently only the case for $\mathrm{Cu}(170)$ and for dominating noble metal surfaces $(169,173)$. Furthermore, "suitable" is based on the assumption that the equilibration at the force field level brings the system into the vicinity of the DFT equilibrium ensemble, a hypothesis that is difficult to (in-)validate in the absence of rigorous DFT based sampling.

From a statistical mechanics point of view, the atomistic description of the interface is meaningless in the absence of a representative phase-space sampling. Typically $\sim 10^{6}$ time steps, corresponding to 1 ns, are necessary to capture entropic effects appropriately. Alternatively, implicit solvation models $(174,175)$ or the in practice basically equivalent(176) classical density functional theory for liquids $(139,177)$ could in principle achieve a computationally much more efficient description of the liquid phase. These methods derive their efficiency from the mean-field treatment, i.e., by averaging 
the fast solvent modes leading to the description of the liquid phase as an effective medium. This is particularly attractive at interfaces, where the diffusion coefficient is low,(178) requiring equilibration times of 0.5 ns. $(169,179)$ Hence, a fundamental questions arises:(180) The electrolyte relaxation times ( $0.5 \mathrm{~ns}$ ) are long compared to individual bond formation times ( $1 \mathrm{ps})$, although fast compared to turn over frequencies where one reactive event per second and active site is generally acceptable. Therefore, comparatively rare electrolyte arrangements that lower the activation barrier could dominate the overall kinetics due to the exponential dependence of the rate on the activation energy, in analogy to the relation between nanoparticle shape and activity.(181) As well appreciated, Marcus theory identifies solvent reorganizations as one of the major contributions to the activation energy of electron transfer processes,(182) i.e., the electron transfer only occurs when the solvent fluctuations have generated a favorable environment. Furthermore, we have shown that the solvation-shell of cations indeed fluctuate significantly, and cation-specifically, at the electrified interface.(183) For electrocatalytic reactions, where both the electrolyte and the adsorbate have to rearrange, the situation is less clear: During the reaction, the adsorbate undergoes a bond-breaking/formation process (horizontal axis of Figure 5) and the electrolyte (vertical axis) has to adapt to the new arrangement. If the solvent rearrangement is fast (flat solvation free energy surface in y-direction) compared to the nuclear reorganization, then the adiabatic approximation applied to the stationary states can also be applied to the transition state (TS) and no special treatment is necessary. If, however, the electrolyte has to re-organize significantly, then such a procedure is inadequate.

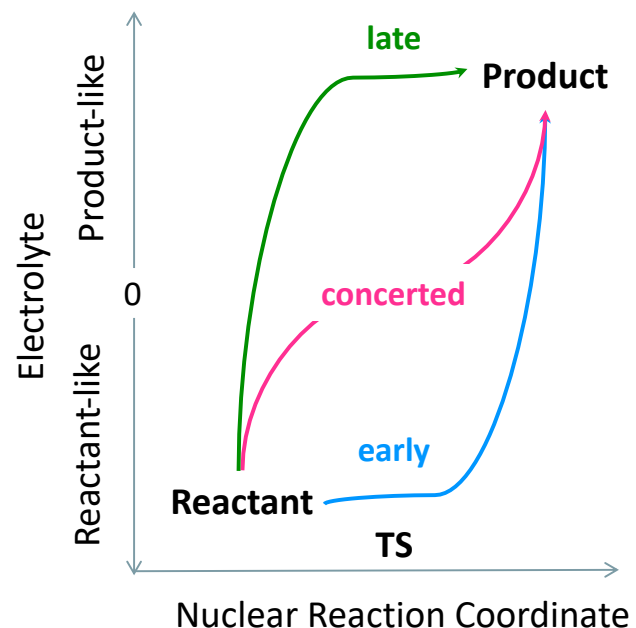

Figure 5 Model Free Energy surface in terms of the adsorbate, nuclear coordinate (x-axis) and the electrolyte arrangement coordinate (y-axis). Early and late transition states with respect to the electrolyte coordinate can be distinguished.

Although non-equilibrium continuum solvation models exist, it is unlikely that they will be able to resolve this fundamental question of coupling between the solution and adsorbate dynamics. The model Hamiltonians of section 3.3.2 are, however, ideally suited to address these questions.(159) For thermodynamic quantities, i.e., reaction energies, the implicit solvation models come with a significant advantage: in contrast with the explicit phase space sampling, which is associated with a statistical uncertainty, the effective medium reproducibly determines a single number. Additionally, electronic structure computations in conjunction with an implicit solvent are associated with only a very small computational overhead compared to vacuum, so that reaction pathways are efficiently and conveniently accessible.(184-186,138) However, these implicit solvation models have several shortcomings: (i) by construction, the implicit solvent and electrolyte will never actively participate in 
the reaction and not even act as a relay for proton transfers. This is likely at the origin of their negligible influence on scaling relations. $(187,163)$ Therefore, such "strong" or "direct" solvation effects need to be modelled via the explicit inclusion of the relevant solvent molecules. $(163,188)$ (ii) Many models, and in particular the ones that belong to the broad class of polarizable continuum models, neglect the size of solvent or electrolyte molecules and ions. As a consequence, the effective medium is in too close contact with the quantum mechanical system. This leads to formally filled cavities and the unphysical presence of solution between weakly bound layers (e.g., in graphite and $\mathrm{MoS}_{2}$ ), even if there is no space to accommodate solvent molecules. $(145,189)$ Strategies to overcome the issue are actively explored.(190) (iii) All of these models reside on more or less extensive parametrization. The existence of comprehensive tables for solvation free energies in bulk solution(191) makes this parametrization rather unproblematic for solvated molecules and ions, even though the crude approximations in implicit solvent models prohibits the development of truly universal models, spurring the continuous development in the field. $(192,193,184,194,186,195)$ Such experimental benchmark data is much more sparse and less diverse for the (electrified) surfaces, so that the current parametrizations are only based on bulk solvation properties of organic molecules and ions. Therefore, while being very attractive from a practical point of view, the accuracy of these models is very questionable. $(196,197)$

\subsection{The electronic structure level of theory: Dominance and Limitation of Density Functional Approximations}

As for any computational investigation using electronic structure methods, the accuracy and cost of the level of theory has to be balanced. For heterogeneous electrocatalysis, the choices are rather limited by seemingly trivial requirement: The electronic structure method needs to be able to describe (periodic) metallic systems. For example, this is not the case for the perturbative Møller-Plesset wave function methods.(198) MP2, which is widely used in main-group chemistry, diverges for gap-less systems. Even the most efficient, (near) linear scaling density matrix optimization algorithms $(199,200)$ for Kohn-Sham density functional theory (DFT),(201) rely on the existence of a fundamental band-gap. Hence, they cannot be applied to metals. To overcome this technical limitation it has been proposed to neglect the partially occupied orbitals altogether.(202) While it technically works after an initialization using a different method, the consequence of simulating this somewhat unphysical electronic structure on the derived properties is hard to predict. At the expense of a very large prefactor, rigorous near-linear scaling can be achieved while preserving the physics of partially occupied orbitals. $(203,204)$ This method only becomes competitive for large systems (> 1000 atoms) and supercomputers, which prohibits its routine application. The second requirement related to the electronic structure method is, of course, its robustness and accuracy. Since heterogeneous (electro)catalysis deals with the activation of small molecules such as $\mathrm{CO}, \mathrm{CO}_{2}, \mathrm{~N}_{2}$ and $\mathrm{O}_{2}$, on surfaces that range from transition metals to oxides, sulfides and TM-doped graphenes, the room for error cancellation is rather limited. Indeed, depending on the interaction of the atoms and molecular fragments with the catalyst, the change in electronic structure is significant.(205) 


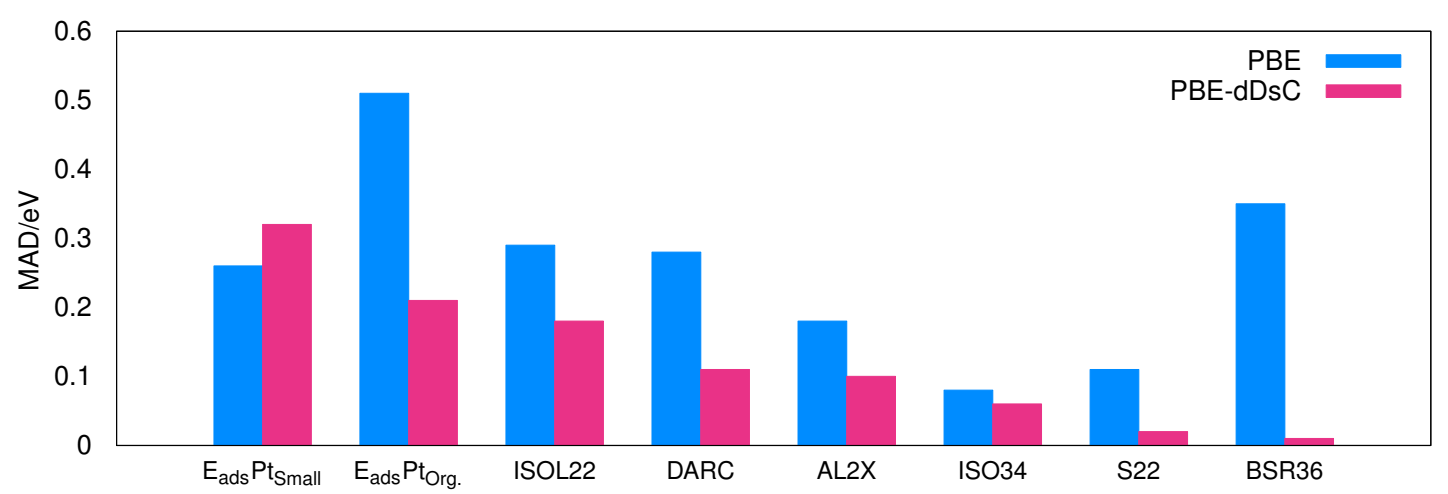

Figure 6 Mean average deviations (MADs) for eight diverse test sets. The performance for PBE (blue) and the dispersion corrected PBE-dDsC (pink) are shown. The data for surface adsorption is taken from ref (206). The $E_{\text {ads }} P t_{\text {small }}$ refers to $H, O$ and CO adsorption on Pt(111). $E_{\text {ads }} P t_{\text {Org. }}$. contains methane, ethane, ethylidyne, cyclohexene, benzene and naphtalene on Pt(111). The other test sets are extracted from GMTKN24 (207). The PBE-dDsC data was published in ref. (208). ISOL22 assesses isomerization energies of large organic molecules. DARC stands for Diels-Alder reaction energies, while AL2X contains dimerization energies of AIX3 species. ISO34 determines isomerization energies of small organic molecules while S22 is dedicated to weak intermolecular interactions. BSR36 measures bond separation energies of alkanes, i.e., hydrogenation reactions of alkanes to methane.

The challenges can be exemplified in the context of the development of density functionals according to the generalized gradient approximation (GGA). PBE,(209) was developed as a general-purpose functional. The functional has then been revised to yield more accurate molecular thermodynamics under the name revPBE.(210) Alternatively, RPBE(211) achieves a better compromise between metallic surfaces and molecular adsorption. Later, PBEsol,(212) was proposed for higher accuracy for solids. Even though these functionals share the philosophy of minimal parameterization, they illustrate the struggles one is facing when having to balance the accuracy of overall (gas-phase) thermodynamics of molecules, the behavior of adsorbates and the electronic structure of solids. Even reproducing the structure and density of liquid water with DFT is challenging,(213) illustrating the additional constraints the liquid component of the solid/liquid interface imposes on the robustness of density functionals for atomistic simulations of electrocatalysis.

The consequences of this limited accuracy are exemplified by the performance for energetic quantities for the few systems where the corresponding benchmark data is well-established. Recently, the claim has appeared that an accuracy of $0.05 \mathrm{eV}$ is routinely achievable in electrocatalysis.(214) However, the solid/gas interface benchmark data suggests that an accuracy of $0.2 \mathrm{eV}(206,215)$ is the best we can hope for in general. This is depicted in Figure 6, where the mono- and diatomic adsorbates on $\mathrm{Pt}(111)$ are separated from the organic adsorbates and typical data for gas-phase thermodynamics is shown as well. The graph also nicely illustrates the benefit of a dispersion correction at the metal/gas interface. Error cancellation or accumulation occurs unpredictably, so that the accuracy for relative energies is expected to be on the same order of $\sim 0.2 \mathrm{eV}$ for surface reaction and adsorption energies, which compares well with the 0.2-0.3 eV gas-phase thermodynamics accuracy for PBE or RPBE.(216) Solution data is much less available, but the accuracy of DFT in solution has no reason to be higher than in gas-phase. Due to the omnipresent delocalization error,(217) the situation at the metal/liquid interface is probably even worse, in particular for electrolyte solutions. Just as a reminder, the delocalization error denotes the observation that semi-local and hybrid density functionals artificially stabilize fractional charges. This shows up as an unphysical smearing of charges among atoms, ions and molecules. The prototypical systems for this fundamental failure are the dissociation limits of alkali metal halides, for which GGAs erroneously predict fractionally charged ions instead of neutral 
atoms.(218) Similarly, the band-alignment at the metal/liquid interface is problematic: The intrinsically delocalized electronic structure of metals leads to generally reasonable predictions for workfunctions by GGAs (within $0.2 \mathrm{eV}$ ).(219) In contrast, the band-gap of semi-conductors and insulators is too small by $40 \%$ on average.(220) As a consequence, the band alignment at the interface can be seriously wrong and leading to spurious results.(221)

\section{RATIONALIZING EXPERIMENTAL HER ACTIVITY WITH ATOMISTIC MODELLING: SUCCESS STORIES}

An ever increasing energy demand due to rapid industrialization has accelerated the need to develop environmentally and economically sustainable means to produce renewable energy. $(1,9,222)$ Hydrogen $\left(\mathrm{H}_{2}\right)$ production from water electrolysis has emerged as a promising process to fuel a carbon-neutral energy economy due to its clean reaction by-products $\left(\mathrm{H}_{2}\right.$ and $\left.\mathrm{O}_{2}\right)$, which can be stored and released in fuel cells on demand.(223) In this regard, significant progress has been made towards industrial-scale water electrolysis for $\mathrm{H}_{2}$ production through combining atomistic modelling and experimental data in the development of efficient and durable hydrogen evolution reaction (HER) electrocatalysts. $(9,48)$ In this section, we review the exemplary progress made by atomistic modelling of electrocatalysis to inform and drive the experimental discovery and optimization of new materials for HER.

Fundamentally, HER in acidic solution proceeds through either of two mechanisms beginning with the Volmer step (Eq. 10), followed by the Heyrovsky (Eq. 11) or Tafel step (Eq. 12). Since both mechanisms begin with the adsorption of a free $\mathrm{H}^{+}$ion in the electrolyte onto an active site on the HER electrocatalyst surface $(*)$, the rate of HER depends on the Gibbs free energy of $\mathrm{H}$ adsorption $\left(\Delta G_{H}\right) \cdot(44,46)$ Using a simple kinetic model, Nørskov et al. established a theoretical correlation between $\Delta G_{H}$ and the HER exchange current density $\left(i_{0}\right)$ for a series of transition metals, in good agreement with the experimentally-obtained volcano plot and Sabatier's principle (Figure 7a).(46) The findings were consistent with platinum $(\mathrm{Pt})$ as the most active pure transition metal HER electrocatalyst due to its near-thermoneutral $\Delta \mathbf{G}_{\mathrm{H}}$, as predicted earlier by Parson.(44) Very recent reports reveal that $\mathrm{Pt}$ is special beyond the $\Delta \mathrm{G}_{\mathrm{H}} \sim 0$ criterion, i.e., that it is the high-coverage differential hydrogen adsorption properties that make Pt particularly active for HER.(224) Nevertheless, the establishment of $\Delta G_{H}$ as a simple yet accurate atomic-scale descriptor for HER catalytic activity has motivated a subsequent computational screening of 736 bimetallic alloy combinations from 16 transition metals. This screening predicted that a BiPt surface alloy could match and potentially outperform Pt in HER activity, given that the calculated $\Delta G_{H}$ difference between Pt and BiPt was $\approx 0.04$ eV.(47) The BiPt alloy was synthesized by annealing its precursor ( $\mathrm{Pt}-\mathrm{B} \mathrm{i}_{\mathrm{ir}}$ ) and its HER performance was evaluated (Figure $\mathbf{7 b}$ ). From Figure $\mathbf{7 b}$, it was evident that at $0 \mathrm{~V}$, BiPt alloy produced at least $50 \%$ more current density than $\mathrm{Pt}$, (47) suggesting that a good atomistic understanding of the HER mechanism can inform high-throughput computational screening and yield accurate predictions of undiscovered HER catalysts.

Volmer step:

Heyrovsky step:

Tafel step:

$$
\mathrm{H}^{+}+\mathrm{e}^{-}+{ }^{*} \rightarrow \mathrm{H}^{*}
$$

Eq. 10

Eq. 11

$$
\mathrm{H}^{*}+\mathrm{H}^{+}+\mathrm{e}^{-} \rightarrow \mathrm{H}_{2}+*
$$$$
2 \mathrm{H}^{*} \rightarrow \mathrm{H}_{2}+2^{*}
$$ 
Beyond transition metals, efforts have also been made in the computational screening and theoryguided discovery of economically viable and Earth-abundant non-platinum group metal HER electrocatalysts for industrial implementation. $(8,125)$ Hinnemann and co-workers calculated the $\Delta G_{H}$ for various $\mathrm{H}$-binding sites in HER-active hydrogenase and nitrogenase enzymes which revealed that $\Delta G_{H}$ was almost thermoneutral in the enzyme's equatorial sulfur (S) sites, expanding the application of $\Delta G_{H}$ descriptor for HER activity from metals to now include enzymes.(225) Recognizing a close similarity in the coordination number and mode between the nitrogenase $S$ active site and $S$ in $2 \mathrm{D}$ $\mathrm{MoS}_{2}$, the authors then verified the HER activity of $\mathrm{MoS}_{2}$ experimentally to demonstrate the theoryguided discovery of $\mathrm{MoS}_{2}$ as promising HER catalysts. $2 \mathrm{H}-\mathrm{MoS}_{2}$ was subsequently determined to be HER active on its edges, (226) prompting follow-up reports in nanostructuring to expose more $2 \mathrm{H}-\mathrm{MoS}_{2}$ edge sites for enhanced HER activity.(227,228,23) Separately, Li et al. evaluated the effect of introducing $S$ vacancies and elastic strain on $\Delta G_{H}$ and electronic band structure of monolayer $2 \mathrm{H}$ $\mathrm{MoS}_{2 .}$ (229) The results indicated that various levels of strain and $\mathrm{S}$ vacancy combinations could theoretically yield $\Delta G_{H}=0$ in $2 \mathrm{H}-M_{0} S_{2}$ (Figure 7c), since both processes separately affect $\mathrm{H}$ binding to fine-tune and optimize $\Delta \mathrm{G}_{\mathrm{H}}$. Indeed, subsequent experimental results confirmed that a combination of both tensile strain and S vacancies (SV-MoS 2 ) significantly enhanced the intrinsic HER activity of $2 \mathrm{H}$ $\mathrm{MoS}_{2}$ (Figure 7d). Based on grand-canonical DFT computations, we have recently shown that the active sites of $\mathrm{MoS}_{2}$ might change in the presence of water, but remain active through a change in mechanism.(145) Apart from vacancy and strain engineering, other research groups have also employed a variety of phase, defect, dopant and interfacial engineering of 2D transition metal dichalcogenides (TMDs) using $\Delta G_{H}$ as a guiding principle for HER activity prediction and catalyst discovery.(230-236)

Most recently, a newly discovered class of 2D transition metal carbides, nitrides and carbonitrides (MXenes) has emerged as promising HER candidates due to their high metallic conductivity, hydrophilicity and solution processibility.(237-240) MXenes possess a general formula of $M_{n+1} X_{n} T_{x}$, where $\mathrm{n}=1-4, \mathrm{M}=$ early-row transition metal such as $\mathrm{Mo}, \mathrm{Ti}, \mathrm{Cr}$ and $\mathrm{V}, \mathrm{X}=\mathrm{C}$ and/or $\mathrm{N}$ and $\mathrm{T}_{x}=$ surface terminations such as $-\mathrm{O},-\mathrm{OH}$ and $-\mathrm{F}$ formed during MXene synthesis.(241-243) Early works reported that different polar $T_{x}$ functional groups altered the electronic structure and band gap of MXenes,(244) which would consequently affect their $\Delta G_{H}$ and predicted HER activity.(245) Seh et al. first demonstrated the HER activity of MXenes experimentally after computing the volcano plot and identifying $\mathrm{Mo}_{2} \mathrm{CT}_{x}$ as a promising HER electrocatalyst (Figure 7e).(246) Unlike $2 \mathrm{H}-\mathrm{MoS}_{2}$ where HER activity was localized only at its edges, the $-\mathrm{O}$ terminated $\mathrm{MXene}$ basal planes of $\mathrm{Mo}_{2} \mathrm{CT}_{x}$ were determined to be HER active, $(246,247)$ garnering significant interest in exploring MXenes as 2D HER electrocatalysts. Subsequently, follow-up work from Handoko et al. reported that tuning the composition of $T_{x}$ terminations on MXene basal planes affected HER activity.(247) By considering the $-F$ coverage on the MXene basal planes, the authors discovered a good agreement between the experimental and theoretical overpotential (calculated from $\Delta G_{H}$ ) at a current density of $-10 \mathrm{~mA} \mathrm{~cm}{ }^{-2}$ for a variety of MXenes (Figure 7f), thus validating the HER atomistic model and $\Delta G_{H}$ as a predictor for HER activity in MXenes. Thereafter, further computational work informed successive MXene designs to enhance HER activity by modulating $\Delta G_{H}$ through adsorption and doping of transition metals $(26,248-251)$ and non-metals $(252,253)$ onto MXene basal planes and through precise $-T_{x}$ control. $(254,255)$ Currently, efforts have also been made in the computational screening of novel MXenes with promising HER activity using $\Delta G_{H}$ as an indicator for targeted experimental synthesis and validation.(256-259) 
In summary, mechanistic insights into the HER mechanism (Volmer, Tafel and Heyrovsky elementary steps) resulted in the discovery of the correlation between $\Delta G_{H}$ and HER activity. Using $\Delta G_{H}$ as a predictor for HER activity has resulted in good agreement with experimental data for varying classes of materials from transition metals to TMDs and MXenes, and informed numerous successful theoryguided experimental designs with significantly enhanced HER activity in acidic solution. We note in passing that the atomistic origin of the $\mathrm{pH}$ dependence of HER remains a vividly debated topic.(260265) With a strong understanding in atomistic modelling, high-throughput computational screening can yield predictions with high levels of accuracy for targeted experimental work and reconcile experimentally observed trends. Coupled with advanced in-situ and operando characterization, these catalytic insights can be extended beyond HER to other more complex reactions such as $\mathrm{CO}_{2}$ and $\mathrm{N}_{2}$ reduction in the future.(266-268)

a

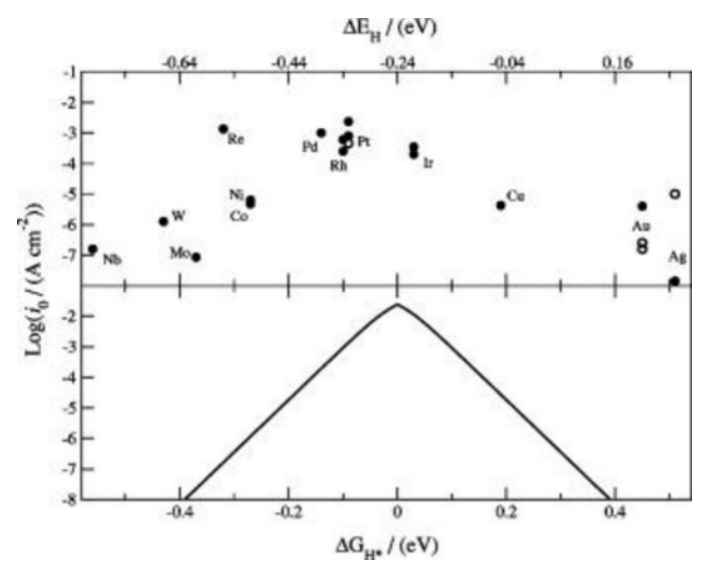

C

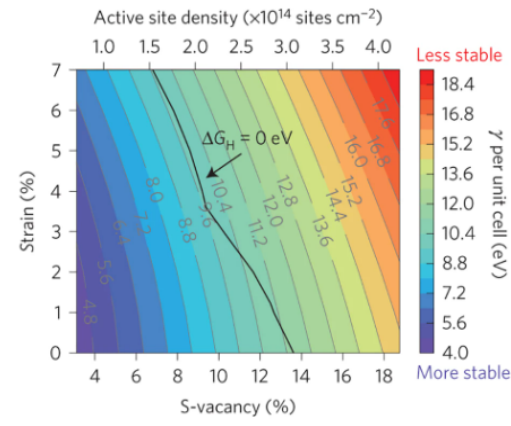

e

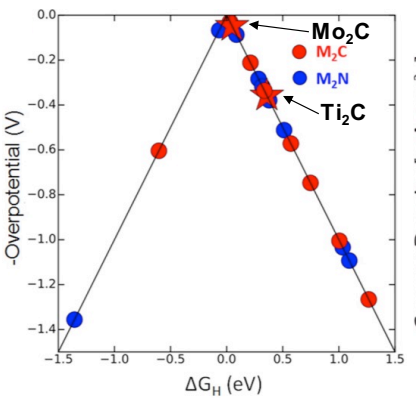

b

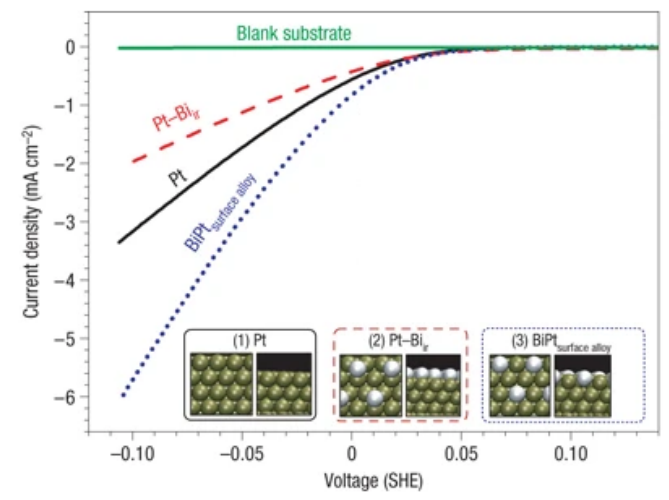

d

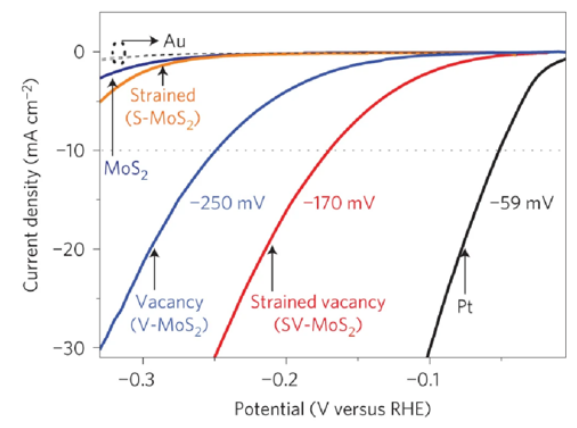

f

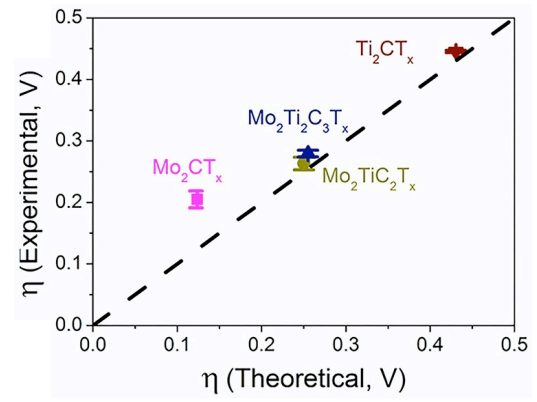

Figure 7 Reconciling theory with experiment results for HER electrocatalysts. (a) (top) Experimental io plotted against theoretically calculated $\Delta G_{H}$ for various transition metal surfaces, compared against the proposed kinetic model (below). Open circles represent single crystal data. Reproduced from(46), copyright IOP science. (b) Experimental HER data of Pt (black), BiPt alloy precursor (Pt-Bi ir, red dash) and the synthesized BiPt alloy (blue dots). Modified from(47), copyright Nature Publishing Group. (c) Colour contour plot of stability (measured by surface energy per unit cell $\gamma$ ) with varying levels of strain and $S$ vacancies. Black line illustrates combinations of strain and $S$ vacancies for $\Delta G_{H}=0$. (d) HER data of Au substrate, Pt control and $2 \mathrm{H}-\mathrm{MoS}_{2}$ (pristine and with various treatments). (c) and (d) reproduced from (229), copyright Nature Publishing 
Group. (e) Theoretical volcano plot of $\mathrm{MXenes}\left(\mathrm{Ti}_{2} \mathrm{C}\right.$ and $\mathrm{Mo}_{2} \mathrm{C}$ marked out with red stars) and HER activity of $\mathrm{Ti}_{2} \mathrm{CT}_{x}$ and $\mathrm{Mo}_{2} \mathrm{CT}_{x}$. Modified from(246), copyright American Chemical Society. (f) Comparison of theoretical and experimental overpotential at $-10 \mathrm{~mA} \mathrm{~cm} \mathrm{~cm}^{-2}$ for various MXenes after considering $-F$ coverage on basal planes. Dashed line indicates complete agreement. Reproduced from(247), copyright American Chemical Society.

\section{VALIDATION: WHAT MORE COULD BE DONE?}

The development of advanced methods for the atomistic modelling of electrocatalysis is thriving. However, it is far from obvious to judge the general applicability and improvement of one approach compared to another. Furthermore, many of the most sophisticated approaches are basically designed ad hoc and used only in a couple of studies, which makes comparisons even harder. If, furthermore, the used software is either not public or system specific (e.g., only for Cu catalysts), the method should not be considered generally validated. From the experimental point of view, validation of theoretical (partial) current densities as a function of the potential are the most attractive quantities to reproduce. Such data is indeed increasingly available through online mass-spectrometry.(269) However, as argued in the introduction, these kinetic quantities necessitate the most complete theoretical models - or rely on the most drastic approximations. Hence, while appealing, these quantities are not the most suitable ones for the validation of the atomistic description of the processes at the interface. In contrast, for few systems the potential dependent atomistic structure of the interface is known. In this respect, EC-STM is most valuable, since it gives a direct depiction of the atomistic structure.(270-275) Alternative operando (potential dependent) spectroscopies give also precious information about surface state under operating conditions.(276,277) Reproducing (and interpreting) such data from first principles brings methods one step further towards validation. Nevertheless, most of these techniques do not directly probe the electrochemical reactivity. For instance, the Stark tuning slopes, which have been and still are, popular to validate theoretical models,(278) probe the local electrostatic field, the geometry of the adsorbate and the IR frequencies, but do not tell anything about the reactivity. The quantification of capacitance as a function of the electrochemical potential assesses, on the other hand, the realism of the description of the interface. Unfortunately, only few examples exist that are unequivocally associated with one single phenomenon, i.e., not affected by specific adsorption. $(279,280)$ Again, while reassuring if well reproduced, the correct description of the capacitance in the double-layer regime does by no means validate the use of a given method to investigate the reaction mechanism. Over the decades, electrochemists have accumulated a fair quantity of potential dependent adsorption energies over single-crystal surfaces, e.g., for alcohols $(281,282)$ halides, $(283,284)$ sulfate, $(285,286)$ phosphate, (287) uracil(288) and benzoic acid.(289) This data has so far not been exploited for method validation, last but not least because it puts stringent requirements on the compatibility and relative reliability of the solvation model and the description of the adsorbate-electrode interaction. This would, however, bring theoretical methods a significant step towards validation, as the description of reversibly bound adsorbates is an integral part of reaction mechanisms. Last but not least, the electrosorption values which are experimentally determined and give information on the exchange of electrons between the adsorbate and the electrode (electron reservoir) give direct access to the (re)distribution of electrons during elementary steps (such as adsorption) and can, therefore, be considered fundamentally important quantities for electrocatalysis.(290) We suggest that a first principles-based method that describes potential dependent geometries, capacities and adsorption energies and electrosorption values quantitatively can be considered validated for the use of the elucidation of reaction mechanisms for electrocatalysis with increasing reliability in this order. 


\section{Conclusion}

In this overview, we have highlighted the challenges that atomistic modelling of electrified interfaces and in particular electrocatalysis is facing. We have described the various methods that are currently applied to gain an atomistic understanding of reaction mechanisms at the electrode surfaces and reviewed the exemplary case of the hydrogen evolution reaction, where the complementary efforts of theory and experiment have proven most fruitful, leading to catalysts based on Earth-abundant materials that rival with the precious platinum benchmark catalysts.

Beyond the standard challenges in heterogenous catalysis which are the limited accuracy of affordable density functional approximations for periodic, metallic systems and the choice of the active site model, the fundamental challenge in the field of first-principles based modelling of electrocatalysis is the difficulty to validate the models: experimentally, kinetic measurements are the most available data. However, between the atomistic picture of the electrode and the macroscopically measured kinetics, there is a long, hazardous connection, which can be seen like a novel: if we want to tell the entire story with all its details in order to provide a true understanding of the electrochemical mechanisms, we have to deploy extraordinary efforts. If, however, we are more interested in screening catalysts, we can skip through all but the first and last pages. These crude approximations are very powerful indeed, as they allow to explore the chemical space efficiently. In this review, we have highlightd the virtues and techniques of the complementary approach, which consists in the characterization of the protagonists (the processes at the electrified interface). We foresee a bright future for methods that combine extensive phase-space sampling with a control of the electrochemical potential. Assuming that the DFT computations provide sufficiently accurate energies for these complex systems, this combination will allow to clarify electrolyte and $\mathrm{pH}$ effects, but will also shed light on the fundamental principles of electrocatalysis, where the role and coupling between adsorbate and electrolyte solution dynamics remains to be uncovered.

\section{Funding Information}

This work was financially supported by Région Auvergne Rhône-Alpes through the project Pack Ambition Recherche 2018 MoSHi. Financial support from the Singapore National Research Foundation (NRF-NRFF2017-04) is acknowledged.

\section{References}

1. Lewis NS, Nocera DG. Powering the planet: Chemical challenges in solar energy utilization. Proc Natl Acad Sci. 2006;103(43):15729-15735.

2. Bockris JO. Hydrogen no longer a high cost solution to global warming: New ideas. Int J Hydrog Energy. 2008;33(9):2129-2131.

3. Seshadri G, Lin C, Bocarsly AB. A new homogeneous electrocatalyst for the reduction of carbon dioxide to methanol at low overpotential. J Electroanal Chem. 1994;372(1):145-50.

4. Yan Y, Zeitler EL, Gu J, Hu Y, Bocarsly AB. Electrochemistry of Aqueous Pyridinium: Exploration of a Key Aspect of Electrocatalytic Reduction of $\mathrm{CO} 2$ to Methanol. J Am Chem Soc. 2013;135(38):14020-3. 
5. Neri G, Donaldson PM, Cowan AJ. The Role of Electrode-Catalyst Interactions in Enabling Efficient $\mathrm{CO}_{2}$ Reduction with $\mathrm{Mo}(\mathrm{bpy})(\mathrm{CO})_{4}$ As Revealed by Vibrational Sum-Frequency Generation Spectroscopy. J Am Chem Soc. 2017;139(39):13791-7.

6. Franco AA, Rucci A, Brandell D, Frayret C, Gaberscek M, Jankowski P, et al. Boosting Rechargeable Batteries R\&D by Multiscale Modeling: Myth or Reality? Chem Rev. 2019;119(7):4569-627.

7. Lautar AK, Hagopian A, Filhol J-S. Modeling interfacial electrochemistry: concepts and tools. Phys Chem Chem Phys. 2020;22:10569-80.

8. Norskov JK, Bligaard T, Rossmeisl J, Christensen $\mathrm{CH}$. Towards the computational design of solid catalysts. Nat Chem. 2009;1(1):37-46.

9. Seh ZW, Kibsgaard J, Dickens CF, Chorkendorff I, Nørskov JK, Jaramillo TF. Combining theory and experiment in electrocatalysis: Insights into materials design. Science. 2017;355(6321):eaad4998.

10. Stampfl C, Veronica Ganduglia-Pirovano M, Reuter K, Scheffler M. Catalysis and corrosion: the theoretical surface-science context. Surf Sci. 2002;500(1):368-94.

11. Taylor CD. Corrosion informatics: an integrated approach to modelling corrosion. Corros Eng Sci Technol. 2015;50(7):490-508.

12. Merola C, Cheng H-W, Schwenzfeier K, Kristiansen K, Chen Y-J, Dobbs HA, et al. In situ nano- to microscopic imaging and growth mechanism of electrochemical dissolution (e.g., corrosion) of a confined metal surface. Proc Natl Acad Sci. 2017;114(36):9541-6.

13. Nace-International-Report.pdf [Internet]. [cited 2019]. Available from: http://impact.nace.org/documents/Nace-International-Report.pdf

14. Heiskanen SK, Kim J, Lucht BL. Generation and Evolution of the Solid Electrolyte Interphase of Lithium-Ion Batteries. Joule. 2019;3(10):2322-33.

15. Kopač Lautar A, Bitenc J, Rejec T, Dominko R, Filhol J-S, Doublet M-L. Electrolyte Reactivity in the Double Layer in Mg Batteries: An Interface Potential-Dependent DFT Study. J Am Chem Soc. 2020;142(11):5146-53.

16. Simon P, Gogotsi Y. Materials for electrochemical capacitors. Nat Mater. 2008;7(11):845-54.

17. Zhan C, Lian C, Zhang Y, Thompson MW, Xie Y, Wu J, et al. Computational Insights into Materials and Interfaces for Capacitive Energy Storage. Adv Sci. 2017;4(7):1700059.

18. Burdyny T, Smith WA. $\mathrm{CO}_{2}$ reduction on gas-diffusion electrodes and why catalytic performance must be assessed at commercially-relevant conditions. Energy Environ Sci. 2019;12(5):1442-53.

19. Laguna-Bercero MA. Recent advances in high temperature electrolysis using solid oxide fuel cells: A review. J Power Sources. 2012;203(0):4-16.

20. Vayenas CG, Bebelis S, Yentekakis IV, Lintz H-G. Non-faradaic electrochemical modification of catalytic activity: A status report. Catal Today. 1992;11(3):303-438. 
21. Pander JE, Baruch MF, Bocarsly AB. Probing the Mechanism of Aqueous CO2 Reduction on Post-Transition-Metal Electrodes using ATR-IR Spectroelectrochemistry. ACS Catal. 2016;6(11):7824-33.

22. Subbaraman R, Tripkovic D, Chang K-C, Strmcnik D, Paulikas AP, Hirunsit P, et al. Trends in activity for the water electrolyser reactions on $3 \mathrm{~d} \mathrm{M}(\mathrm{Ni}, \mathrm{Co}, \mathrm{Fe}, \mathrm{Mn})$ hydr(oxy)oxide catalysts. Nat Mater. 2012;11(6):550-7.

23. Benck JD, Hellstern TR, Kibsgaard J, Chakthranont P, Jaramillo TF. Catalyzing the Hydrogen Evolution Reaction (HER) with Molybdenum Sulfide Nanomaterials. ACS Catal. 2014;4(11):3957-71.

24. Yu P, Wang F, Shifa TA, Zhan X, Lou X, Xia F, et al. Earth abundant materials beyond transition metal dichalcogenides: A focus on electrocatalyzing hydrogen evolution reaction. Nano Energy. 2019;58:244-76.

25. Dasgupta A, Rioux RM. Intermetallics in catalysis: An exciting subset of multimetallic catalysts. Catal Today. 2019;330:2-15.

26. Handoko AD, Steinmann SN, Seh ZW. Theory-guided materials design: two-dimensional MXenes in electro- and photocatalysis. Nanoscale Horiz. 2019;4(4):809-27.

27. Wu G, Zelenay P. Nanostructured Nonprecious Metal Catalysts for Oxygen Reduction Reaction. Acc Chem Res. 2013;46(8):1878-89.

28. Masa J, Xia W, Muhler M, Schuhmann W. On the Role of Metals in Nitrogen-Doped Carbon Electrocatalysts for Oxygen Reduction. Angew Chem Int Ed. 2015;54(35):10102-20.

29. Leung K. DFT modelling of explicit solid-solid interfaces in batteries: methods and challenges. Phys Chem Chem Phys. 2020;22:10412-25.

30. Wu C, Schmidt DJ, Wolverton C, Schneider WF. Accurate coverage-dependence incorporated into first-principles kinetic models: Catalytic NO oxidation on Pt (111). J Catal. 2012;286:8894.

31. Reuter K. Ab Initio Thermodynamics and First-Principles Microkinetics for Surface Catalysis. Catal Lett. 2016;146(3):541-563.

32. Stamenkovic VR, Strmcnik D, Lopes PP, Markovic NM. Energy and fuels from electrochemical interfaces. Nat Mater. 2017;16(1):57-69.

33. Ong BC, Kamarudin SK, Basri S. Direct liquid fuel cells: A review. Int J Hydrog Energy. 2017;42(15):10142-57.

34. Zhu DD, Liu JL, Qiao SZ. Recent Advances in Inorganic Heterogeneous Electrocatalysts for Reduction of Carbon Dioxide. Adv Mater. 2016;28(18):3423-52.

35. Cui X, Tang C, Zhang Q. A Review of Electrocatalytic Reduction of Dinitrogen to Ammonia under Ambient Conditions. Adv Energy Mater. 2018;8(22):1800369.

36. Steinmann SN, Michel C, Schwiedernoch R, Wu M, Sautet P. Electro-carboxylation of butadiene and ethene over Pt and Ni catalysts. J Catal. 2016;343:240. 
37. Wang C-Y. Fundamental Models for Fuel Cell Engineering. Chem Rev. 2004;104(10):4727-4766.

38. Weber AZ, Newman J. Modeling Transport in Polymer-Electrolyte Fuel Cells. Chem Rev. 2004;104(10):4679-4726.

39. Bernardi DM, Verbrugge MW. A Mathematical Model of the Solid-Polymer-Electrolyte Fuel Cell. J Electrochem Soc. 1992;139(9):2477-2491.

40. Jahnke T, Futter G, Latz A, Malkow T, Papakonstantinou G, Tsotridis G, et al. Performance and degradation of Proton Exchange Membrane Fuel Cells: State of the art in modeling from atomistic to system scale. J Power Sources. 2016;304:207-33.

41. Costentin C, Savéant J-M, Tard C. Catalysis of $\mathrm{CO}_{2}$ Electrochemical Reduction by Protonated Pyridine and Similar Molecules. Useful Lessons from a Methodological Misadventure. ACS Energy Lett. 2018;3(3):695-703.

42. Quaino $P$, Juarez $F$, Santos $E$, Schmickler $W$. Volcano plots in hydrogen electrocatalysis - uses and abuses. Beilstein J Nanotechnol. 2014;5(1):846-54.

43. Sabatier F. La catalyse en chimie organique. Paris: Berauge; 1920.

44. Parsons R. The rate of electrolytic hydrogen evolution and the heat of adsorption of hydrogen. Trans Faraday Soc. 1958;54(0):1053-63.

45. Trasatti S. Work function, electronegativity, and electrochemical behaviour of metals: III. Electrolytic hydrogen evolution in acid solutions. J Electroanal Chem Interfacial Electrochem. 1972;39(1):163-84.

46. Nørskov JK, Bligaard T, Logadottir A, Kitchin JR, Chen JG, Pandelov S, et al. Trends in the Exchange Current for Hydrogen Evolution. J Electrochem Soc. 2005;152(3):J23.

47. Greeley J, Jaramillo TF, Bonde J, Chorkendorff I, Norskov JK. Computational high-throughput screening of electrocatalytic materials for hydrogen evolution. Nat Mater. 2006;5(11):909913.

48. Norskov JK, Christensen $\mathrm{CH}$. Toward Efficient Hydrogen Production at Surfaces. Science. 2006;312(5778):1322-3.

49. Monyoncho EA, Steinmann SN, Sautet P, Baranova EA, Michel C. Computational screening for selective catalysts: Cleaving the $\mathrm{CC}$ bond during ethanol electro-oxidation reaction. Electrochimica Acta. 2018;274:274-8.

50. Abild-Pedersen F, Greeley J, Studt F, Rossmeisl J, Munter TR, Moses PG, et al. Scaling Properties of Adsorption Energies for Hydrogen-Containing Molecules on Transition-Metal Surfaces. Phys Rev Lett. 2007;99(1):016105.

51. Montemore MM, Medlin JW. Scaling relations between adsorption energies for computational screening and design of catalysts. Catal Sci Technol. 2014;4(11):3748-61.

52. Calle-Vallejo F, Loffreda D, Koper MTM, Sautet P. Introducing structural sensitivity into adsorption-energy scaling relations by means of coordination numbers. Nat Chem. 2015;7(5):403-410. 
53. Vojvodic A, Nørskov JK. New design paradigm for heterogeneous catalysts. Natl Sci Rev. 2015;2(2):140-3.

54. Montoya JH, Seitz LC, Chakthranont P, Vojvodic A, Jaramillo TF, Nørskov JK. Materials for solar fuels and chemicals. Nat Mater. 2017;16(1):70-81.

55. Pérez-Ramírez J, López N. Strategies to break linear scaling relationships. Nat Catal. 2019;2(11):971-6.

56. Fletcher S. Tafel slopes from first principles. J Solid State Electrochem. 2009;13(4):537-49.

57. Gnanamuthu DS, Petrocelli JV. A Generalized Expression for the Tafel Slope and the Kinetics of Oxygen Reduction on Noble Metals and Alloys. J Electrochem Soc. 1967;114(10):1036.

58. Holewinski A, Linic S. Elementary Mechanisms in Electrocatalysis: Revisiting the ORR Tafel Slope. J Electrochem Soc. 2012;159(11):H864.

59. Shinagawa T, Garcia-Esparza AT, Takanabe K. Insight on Tafel slopes from a microkinetic analysis of aqueous electrocatalysis for energy conversion. Sci Rep. 2015;5(1):13801.

60. Norskov JK, Rossmeisl J, Logadottir A, Lindqvist L, Kitchin JR, Bligaard T, et al. Origin of the Overpotential for Oxygen Reduction at a Fuel-Cell Cathode. J Phys Chem B. 2004;108(46):17886-17892.

61. Koper MTM, Lukkien JJ, Jansen APJ, van Santen RA. Lattice Gas Model for CO Electrooxidation on Pt-Ru Bimetallic Surfaces. J Phys Chem B. 1999;103(26):5522-9.

62. Chun H-J, Apaja V, Clayborne A, Honkala K, Greeley J. Atomistic Insights into Nitrogen-Cycle Electrochemistry: A Combined DFT and Kinetic Monte Carlo Analysis of NO Electrochemical Reduction on Pt(100). ACS Catal. 2017;7(6):3869-82.

63. Rossmeisl J, Logadottir A, Norskov JK. Electrolysis of water on (oxidized) metal surfaces. Chem Phys. 2005;319(1-3):178-184.

64. Curutchet A, Colinet P, Michel C, Steinmann SN, Bahers TL. Two-sites are better than one: revisiting the OER mechanism on $\mathrm{CoOOH}$ by DFT with electrode polarization. Phys Chem Chem Phys. 2020;22(13):7031-8.

65. Exner KS, Sohrabnejad-Eskan I, Over H. A Universal Approach To Determine the Free Energy Diagram of an Electrocatalytic Reaction. ACS Catal. 2018;8(3):1864-79.

66. Exner KS. Is Thermodynamics a Good Descriptor for the Activity? Re-Investigation of Sabatier's Principle by the Free Energy Diagram in Electrocatalysis. ACS Catal. 2019;5320-9.

67. Exner KS. Design Criteria for Oxygen Evolution Electrocatalysts from First Principles: Introduction of a Unifying Material-Screening Approach. ACS Appl Energy Mater. 2019;2(11):7991-8001.

68. Bockris JO. Overpotential. A lacuna in scientific knowledge. J Chem Educ. 1971;48(6):352.

69. Anderson AB. Molecular orbital theory for catalysis. Structures, energy levels, and reactions of acetylene with $\mathrm{Ni2}(\mathrm{COD}) 2$ (RC.tplbond.CR), Ni2(C5H5)2(RC.tplbond.CR), and the nickel(111) surface. J Am Chem Soc. 1978;100(4):1153-9. 
70. Anderson $\mathrm{AB}$, Kötz $\mathrm{R}$, Yeager $\mathrm{E}$. Theory for $\mathrm{C}-\mathrm{N}-$ and $\mathrm{Ag}-\mathrm{C}$ vibrational frequency dependence on potential: cyanide on a silver electrode. Chem Phys Lett. 1981;82(1):130-4.

71. Anderson AB, Ray NK. Structures and reactions of hydronium, water, and hydroxyl on an iron electrode. Potential dependence. J Phys Chem. 1982;86(4):488-94.

72. Nakatsuji H. Dipped adcluster model for chemisorptions and catalytic reactions on a metal surface. J Chem Phys. 1987;87(8):4995-5001.

73. Santos $E$, Schmickler W. Changes in the surface energy during the reconstruction of $\mathrm{Au}(100)$ and $\mathrm{Au}(111)$ electrodes. Chem Phys Lett. 2004;400(1):26-9.

74. Bureau C, Lécayon G. On a modeling of voltage-application to metallic electrodes using density functional theory. J Chem Phys. 1997;106(21):8821-9.

75. Anderson AB, Kang DB. Quantum Chemical Approach to Redox Reactions Including Potential Dependence: Application to a Model for Hydrogen Evolution from Diamond. J Phys Chem A. 1998;102(29):5993-6.

76. Khan SUM, Bockris JO. Electronic states in solution and charge transfer. J Phys Chem. 1983;87(14):2599-603.

77. Anderson AB, Albu TV. Ab Initio Determination of Reversible Potentials and Activation Energies for Outer-Sphere Oxygen Reduction to Water and the Reverse Oxidation Reaction. J Am Chem Soc. 1999;121(50):11855-63.

78. Anderson AB, Albu TV. Catalytic Effect of Platinum on Oxygen Reduction An Ab Initio Model Including Electrode Potential Dependence. J Electrochem Soc. 2000;147(11):4229-4238.

79. Bureau C, Kranias S, Crispin X, Bredas J-L. DFT Modeling of Stark-Tuning Effect: CO on Polarized $\mathrm{Pd}(100)$ as a Probe for Double-Layer Electrostatic Effects in Electrochemistry. In: HernándezLaguna A, Maruani J, McWeeny R, Wilson S, editors. Quantum Systems in Chemistry and Physics Volume 2: Advanced Problems and Complex Systems Granada, Spain, 1998.

Dordrecht: Springer Netherlands; 2000. p. 169-92. (Progress in Theoretical Chemistry and Physics).

80. Crispin X, Geskin VM, Bureau C, Lazzaroni R, Schmickler W, Brédas JL. A density functional model for tuning the charge transfer between a transition metal electrode and a chemisorbed molecule via the electrode potential. J Chem Phys. 2001;115(22):10493-9.

81. Koper MTM, van Santen RA, Wasileski SA, Weaver MJ. Field-dependent chemisorption of carbon monoxide and nitric oxide on platinum-group (111) surfaces: Quantum chemical calculations compared with infrared spectroscopy at electrochemical and vacuum-based interfaces. J Chem Phys. 2000;113(10):4392-407.

82. Liu P, Logadottir A, Nørskov JK. Modeling the electro-oxidation of $\mathrm{CO}$ and $\mathrm{H} 2 / \mathrm{CO}$ on $\mathrm{Pt}, \mathrm{Ru}$, PtRu and Pt3Sn. Electrochimica Acta. 2003;48(25-26):3731-42.

83. Vuilleumier R, Sprik M, Alavi A. Computation of electronic chemical potentials using free energy density functionals. J Mol Struct. 2000;506:11.

84. Lozovoi AY, Alavi A, Kohanoff J, Lynden-Bell RM. Ab initio simulation of charged slabs at constant chemical potential. J Chem Phys. 2001;115(4):1661-9. 
85. Lozovoi AY, Alavi A. Reconstruction of charged surfaces: General trends and a case study of Pt(110) and Au(110). Phys Rev B. 2003;68(24):245416-.

86. Blumberger J, Bernasconi L, Tavernelli I, Vuilleumier R, Sprik M. Electronic Structure and Solvation of Copper and Silver lons: A Theoretical Picture of a Model Aqueous Redox Reaction. J Am Chem Soc. 2004;126(12):3928-38.

87. Tateyama Y, Blumberger J, Sprik M, Tavernelli I. Density-functional molecular-dynamics study of the redox reactions of two anionic, aqueous transition-metal complexes. J Chem Phys. 2005;122(23):234505.

88. Anderson AB. Theory at the electrochemical interface: reversible potentials and potentialdependent activation energies. Electrochimica Acta. 2003;48(25):3743-9.

89. Roques $A B$ Jérôme; Anderson. Theory for the Potential Shift for OHads Formation on the Pt Skin on Pt3Cr(111) in Acid. J Electrochem Soc. 2004;151(3):E85.

90. Roques J, Anderson AB. Electrode Potential-Dependent Stages in OHads Formation on the Pt3Cr Alloy (111) Surface. J Electrochem Soc. 2004;151:E340.

91. Sha Y, Yu TH, Liu Y, Merinov BV, Goddard WA. Theoretical Study of Solvent Effects on the Platinum-Catalyzed Oxygen Reduction Reaction. J Phys Chem Lett. 2010;1(5):856-861.

92. Sakong S, Naderian M, Mathew K, Hennig RG, Groß A. Density functional theory study of the electrochemical interface between a Pt electrode and an aqueous electrolyte using an implicit solvent method. J Chem Phys. 2015;142(23):234107.

93. Stamenkovic V, Mun BS, Mayrhofer KJJ, Ross PN, Markovic NM, Rossmeisl J, et al. Changing the Activity of Electrocatalysts for Oxygen Reduction by Tuning the Surface Electronic Structure. Angew Chem Int Ed. 2006;45(18):2897-2901.

94. Morgan BJ, Watson GW. A DFT+U description of oxygen vacancies at the TiO2 rutile (110) surface. Surf Sci. 2007;601(21):5034-41.

95. Kuo T-J, Huang MH. Gold-Catalyzed Low-Temperature Growth of Cadmium Oxide Nanowires by Vapor Transport. J Phys Chem B. 2006;110(28):13717-21.

96. Nilekar AU, Mavrikakis M. Improved oxygen reduction reactivity of platinum monolayers on transition metal surfaces. Surf Sci. 2008;602(14):L89-94.

97. Man IC, Su H-Y, Calle-Vallejo F, Hansen HA, Martinez JI, Inoglu NG, et al. Universality in Oxygen Evolution Electrocatalysis on Oxide Surfaces. ChemCatChem. 2011;3(7):1159-1165.

98. Song G-L, Unocic KA. The anodic surface film and hydrogen evolution on Mg. Corros Sci. 2015;98:758-65.

99. Liu Y, Liu S, Che Z, Zhao S, Sheng X, Han M, et al. Concave octahedral Pd@PdPt electrocatalysts integrating core-shell, alloy and concave structures for high-efficiency oxygen reduction and hydrogen evolution reactions. J Mater Chem A. 2016;4(42):16690-7.

100. Ekspong J, Sharifi T, Shchukarev A, Klechikov A, Wågberg T, Gracia-Espino E. Stabilizing Active Edge Sites in Semicrystalline Molybdenum Sulfide by Anchorage on Nitrogen-Doped Carbon Nanotubes for Hydrogen Evolution Reaction. Adv Funct Mater. 2016;26(37):6766-76. 
101. Karamad M, Hansen HA, Rossmeisl J, Nørskov JK. Mechanistic Pathway in the Electrochemical Reduction of CO2 on RuO2. ACS Catal. 2015;5(7):4075-81.

102. Chen LD, Urushihara M, Chan K, Nørskov JK. Electric Field Effects in Electrochemical CO2 Reduction. ACS Catal. 2016;6(10):7133-9.

103. Liu S, Yang H, Huang X, Liu L, Cai W, Gao J, et al. Identifying Active Sites of Nitrogen-Doped Carbon Materials for the CO2 Reduction Reaction. Adv Funct Mater. 2018;28(21):1800499.

104. Montoya JH, Tsai C, Vojvodic A, Nørskov JK. The Challenge of Electrochemical Ammonia Synthesis: A New Perspective on the Role of Nitrogen Scaling Relations. ChemSusChem. 2015;8(13):2180-6.

105. Choi C, Back S, Kim N-Y, Lim J, Kim Y-H, Jung Y. Suppression of Hydrogen Evolution Reaction in Electrochemical N2 Reduction Using Single-Atom Catalysts: A Computational Guideline. ACS Catal. 2018;8(8):7517-25.

106. Kwon Y, Lai SCS, Rodriguez P, Koper MTM. Electrocatalytic Oxidation of Alcohols on Gold in Alkaline Media: Base or Gold Catalysis? J Am Chem Soc. 2011;133(18):6914-7.

107. Calle-Vallejo F, Koper MTM. Theoretical Considerations on the Electroreduction of $\mathrm{CO}$ to $\mathrm{C}_{2}$ Species on Cu(100) Electrodes. Angew Chem Int Ed. 2013;52(28):7282-5.

108. Katsounaros I, Chen T, Gewirth AA, Markovic NM, Koper MTM. Evidence for Decoupled Electron and Proton Transfer in the Electrochemical Oxidation of Ammonia on Pt(100). J Phys Chem Lett. 2016;7(3):387-92.

109. Goodpaster JD, Bell AT, Head-Gordon M. Identification of Possible Pathways for C-C Bond Formation during Electrochemical Reduction of $\mathrm{CO}_{2}$ : New Theoretical Insights from an Improved Electrochemical Model. J Phys Chem Lett. 2016;7(8):1471-7.

110. Nie X, Esopi MR, Janik MJ, Asthagiri A. Selectivity of CO2 Reduction on Copper Electrodes: The Role of the Kinetics of Elementary Steps. Angew Chem Int Ed. 2013;52(9):2459-2462.

111. Fang Y-H, Wei G-F, Liu Z-P. Constant-Charge Reaction Theory for Potential-Dependent Reaction Kinetics at the Solid-Liquid Interface. J Phys Chem C. 2014;118(7):3629-3635.

112. Chan K, Nørskov JK. Electrochemical Barriers Made Simple. J Phys Chem Lett. 2015;6(14):26638.

113. Steinmann SN, Michel C, Schwiedernoch R, Filhol J-S, Sautet P. Modeling the $\mathrm{HCOOH} / \mathrm{CO} 2$ Electrocatalytic Reaction: When Details Are Key. ChemPhysChem. 2015;16(11):2307-2311.

114. Taylor CD, Wasileski SA, Filhol J-S, Neurock M. First principles reaction modeling of the electrochemical interface: Consideration and calculation of a tunable surface potential from atomic and electronic structure. Phys Rev B. 2006;73(16):165402-.

115. Filhol J-S, Neurock M. Elucidation of the Electrochemical Activation of Water over Pd by First Principles. Angew Chem Int Ed. 2006;45(3):402-406.

116. Taylor C, Kelly RG, Neurock M. First-Principles Calculations of the Electrochemical Reactions of Water at an Immersed Ni(111)/H2O Interface. J Electrochem Soc. 2006;153(12):E207-14. 
117. Lespes N, Filhol J-S. Using Implicit Solvent in Ab Initio Electrochemical Modeling: Investigating Li+/Li Electrochemistry at a Li/Solvent Interface. J Chem Theory Comput. 2015;11(7):33753382.

118. Steinmann SN, Michel C, Schwiedernoch R, Sautet P. Impacts of electrode potentials and solvents on the electroreduction of $\mathrm{CO} 2$ : a comparison of theoretical approaches. Phys Chem Chem Phys. 2015;17(21):13949-63.

119. Otani M, Sugino O. First-principles calculations of charged surfaces and interfaces: A planewave nonrepeated slab approach. Phys Rev B. 2006;73(11):115407-.

120. Otani M, Hamada I, Sugino O, Morikawa Y, Okamoto Y, Ikeshoji T. Electrode Dynamics from First Principles. J Phys Soc Jpn. 2008;77(2):024802.

121. Bonnet N, Morishita T, Sugino O, Otani M. First-Principles Molecular Dynamics at a Constant Electrode Potential. Phys Rev Lett. 2012;109(26):266101-.

122. Haruyama J, Ikeshoji T, Otani M. Electrode potential from density functional theory calculations combined with implicit solvation theory. Phys Rev Mater. 2018;2(9):095801.

123. Rossmeisl J, Norskov JK, Taylor CD, Janik MJ, Neurock M. Calculated Phase Diagrams for the Electrochemical Oxidation and Reduction of Water over Pt(111). J Phys Chem B. 2006;110(43):21833-21839.

124. Rossmeisl J, Skulason E, Bjorketun ME, Tripkovic V, Norskov JK. Modeling the electrified solidliquid interface. Chem Phys Lett. 2008;466(1-3):68-71.

125. Skúlason E, Tripkovic V, Björketun ME, Gudmundsdóttir S, Karlberg G, Rossmeisl J, et al. Modeling the Electrochemical Hydrogen Oxidation and Evolution Reactions on the Basis of Density Functional Theory Calculations. J Phys Chem C. 2010;114(42):18182-97.

126. Hansen MH, Rossmeisl J. pH in Grand Canonical Statistics of an Electrochemical Interface. J Phys Chem C. 2016;120(51):29135-43.

127. Hansen $\mathrm{MH}$, Nilsson $\mathrm{A}$, Rossmeisl J. Modelling $\mathrm{pH}$ and potential in dynamic structures of the water/Pt(111) interface on the atomic scale. Phys Chem Chem Phys. 2017;19(34):23505-14.

128. Cohen AJ, Mori-Sánchez P. Dramatic changes in electronic structure revealed by fractionally charged nuclei. J Chem Phys. 2014;140(4):044110.

129. Chen Y, Roux B. Constant-pH Hybrid Nonequilibrium Molecular Dynamics-Monte Carlo Simulation Method. J Chem Theory Comput. 2015;11(8):3919-31.

130. Radak BK, Chipot C, Suh D, Jo S, Jiang W, Phillips JC, et al. Constant-pH Molecular Dynamics Simulations for Large Biomolecular Systems. J Chem Theory Comput. 2017;13(12):5933-44.

131. Bagger A, Arán-Ais RM, Halldin Stenlid J, Campos dos Santos E, Arnarson L, Degn Jensen K, et al. $\mathrm{Ab}$ Initio Cyclic Voltammetry on $\mathrm{Cu}(111), \mathrm{Cu}(100)$ and $\mathrm{Cu}(110)$ in Acidic, Neutral and Alkaline Solutions. ChemPhysChem. 2019;20(22):3096-105.

132. Jinnouchi R, Anderson AB. Electronic structure calculations of liquid-solid interfaces: Combination of density functional theory and modified Poisson-Boltzmann theory. Phys Rev B. 2008;77(24):245417-. 
133. Fang Y-H, Liu Z-P. Mechanism and Tafel Lines of Electro-Oxidation of Water to Oxygen on RuO2(110). J Am Chem Soc. 2010;132(51):18214-18222.

134. Letchworth-Weaver K, Arias TA. Joint density functional theory of the electrode-electrolyte interface: Application to fixed electrode potentials, interfacial capacitances, and potentials of zero charge. Phys Rev B. 2012;86(7):075140.

135. Ringe S, Oberhofer H, Hille C, Matera S, Reuter K. Function-Space-Based Solution Scheme for the Size-Modified Poisson-Boltzmann Equation in Full-Potential DFT. J Chem Theory Comput. 2016;12(8):4052-4066.

136. Fisicaro G, Genovese L, Andreussi O, Marzari N, Goedecker S. A generalized Poisson and Poisson-Boltzmann solver for electrostatic environments. J Chem Phys. 2016;144(1):014103.

137. Sundararaman R, Goddard WA, Arias TA. Grand canonical electronic density-functional theory: Algorithms and applications to electrochemistry. J Chem Phys. 2017;146(11):114104.

138. Mathew K, Kolluru VSC, Mula S, Steinmann SN, Hennig RG. Implicit self-consistent electrolyte model in plane-wave density-functional theory. J Chem Phys. 2019;151(23):234101.

139. Petrosyan SA, Rigos AA, Arias TA. Joint Density-Functional Theory: Ab Initio Study of Cr2O3 Surface Chemistry in Solution. J Phys Chem B. 2005;109(32):15436-15444.

140. Gunceler D, Letchworth-Weaver K, Sundararaman R, Schwarz KA, Arias TA. The importance of nonlinear fluid response in joint density-functional theory studies of battery systems. Modell Simul Mater Sci Eng. 2013;21(7):074005.

141. Melander MM, Kuisma MJ, Christensen TEK, Honkala K. Grand-canonical approach to density functional theory of electrocatalytic systems: Thermodynamics of solid-liquid interfaces at constant ion and electrode potentials. J Chem Phys. 2018;150(4):041706.

142. Kastlunger $\mathrm{G}$, Lindgren $P$, Peterson AA. Controlled-Potential Simulation of Elementary Electrochemical Reactions: Proton Discharge on Metal Surfaces. J Phys Chem C. 2018;122(24):12771-81.

143. Steinmann SN, Sautet P. Assessing a First-Principles Model of an Electrochemical Interface by Comparison with Experiment. J Phys Chem C. 2016;120(10):5619-5623.

144. Shang R, Steinmann SN, Xu B-Q, Sautet P. Mononuclear Fe in N-doped carbon: computational elucidation of active sites for electrochemical oxygen reduction and oxygen evolution reactions. Catal Sci Technol. 2020;10(4):1006-14.

145. Abidi N, Bonduelle-Skrzypczak A, Steinmann SN. Revisiting the active sites at the MoS2/H2O interface via grand-canonical DFT: The role of water dissociation. ACS Appl Mater Interfaces [Internet]. 2020 [cited 2020]; Available from: https://doi.org/10.1021/acsami.0c06489

146. Hajar YM, Treps L, Michel C, Baranova EA, Steinmann SN. Theoretical insight into the origin of the electrochemical promotion of ethylene oxidation on ruthenium oxide. Catal Sci Technol. 2019;9(21):5915-26.

147. Panaritis C, Michel C, Couillard M, Baranova EA, Steinmann SN. Elucidating the role of electrochemical polarization on the selectivity of the $\mathrm{CO} 2$ hydrogenation reaction over $\mathrm{Ru}$. Electrochimica Acta. 2020;350:136405. 
148. Wang P, Steinmann SN, Fu G, Michel C, Sautet P. Key Role of Anionic Doping for H2 Production from Formic Acid on Pd(111). ACS Catal. 2017;7(3):1955-9.

149. Lluch JM. Perspective on "On the theory of oxidation-reduction reactions involving electron transfer. I." In: Cramer CJ, Truhlar DG, editors. Theoretical Chemistry Accounts: New Century Issue. Berlin, Heidelberg: Springer; 2001. p. 231-3.

150. Hush NS. Adiabatic Rate Processes at Electrodes. I. Energy-Charge Relationships. J Chem Phys. 1958;28(5):962-72.

151. Kornyshev AA, Vilfan I. Phase transitions at the electrochemical interface. Electrochimica Acta. 1995;40(1):109-27.

152. Santos E, Koper MTM, Schmickler W. A model for bond-breaking electron transfer at metal electrodes. Chem Phys Lett. 2006;419(4):421-5.

153. Santos E, Schmickler W. Fundamental aspects of electrocatalysis. Chem Phys. 2007;332(1):3947.

154. Santos E, Koper MTM, Schmickler W. Bond-breaking electron transfer of diatomic reactants at metal electrodes. Chem Phys. 2008;344(1):195-201.

155. Anderson PW. Localized Magnetic States in Metals. Phys Rev. 1961;124(1):41-53.

156. Newns DM. Self-Consistent Model of Hydrogen Chemisorption. Phys Rev. 1969;178(3):112335.

157. Santos E, Lundin A, Pötting K, Quaino P, Schmickler W. Model for the electrocatalysis of hydrogen evolution. Phys Rev B. 2009;79(23):235436.

158. Staub R, lannuzzi M, Khaliullin RZ, Steinmann SN. Energy Decomposition Analysis for Metal Surface-Adsorbate Interactions by Block Localized Wave Functions. J Chem Theory Comput. 2019;15(1):265-75.

159. Santos E, Quaino P, Schmickler W. Theory of electrocatalysis: hydrogen evolution and more. Phys Chem Chem Phys. 2012;14(32):11224.

160. Marcus RA, Siders P. Theory of highly exothermic electron transfer reactions. J Phys Chem. 1982;86(5):622-630.

161. Mills JN, McCrum IT, Janik MJ. Alkali cation specific adsorption onto $\mathrm{fcc}(111)$ transition metal electrodes. Phys Chem Chem Phys. 2014;16(27):13699-707.

162. Saleheen M, Heyden A. Liquid-Phase Modeling in Heterogeneous Catalysis. ACS Catal. 2018;8(3):2188-94.

163. Schweitzer B, Steinmann SN, Michel C. Can microsolvation effects be estimated from vacuum computations? A case-study of alcohol decomposition at the H2O/Pt(111) interface. Phys Chem Chem Phys. 2019;21(10):5368-77.

164. Cantu DC, Padmaperuma AB, Nguyen M-T, Akhade SA, Yoon Y, Wang Y-G, et al. A Combined Experimental and Theoretical Study on the Activity and Selectivity of the Electrocatalytic Hydrogenation of Aldehydes. ACS Catal. 2018;8(8):7645-58. 
165. Sakong S, Forster-Tonigold K, Gross A. The structure of water at a Pt(111) electrode and the potential of zero charge studied from first principles. J Chem Phys. 2016;144(19):194701.

166. Bellarosa L, Garcia-Muelas R, Revilla-Lopez G, Lopez N. Diversity at the Water-Metal Interface: Metal, Water Thickness, and Confinement Effects. ACS Cent Sci. 2016;2(2):109-116.

167. Le J, lannuzzi M, Cuesta A, Cheng J. Determining Potentials of Zero Charge of Metal Electrodes versus the Standard Hydrogen Electrode from Density-Functional-Theory-Based Molecular Dynamics. Phys Rev Lett. 2017;119(1):016801.

168. Cheng $\mathrm{T}$, Xiao $\mathrm{H}$, Goddard WA. Full atomistic reaction mechanism with kinetics for $\mathrm{CO}$ reduction on $\mathrm{Cu}(100)$ from ab initio molecular dynamics free-energy calculations at $298 \mathrm{~K}$. Proc Natl Acad Sci. 2017;114(8):1795-1800.

169. Steinmann SN, Ferreira De Morais R, Götz AW, Fleurat-Lessard P, lannuzzi M, Sautet $P$, et al. Force Field for Water over Pt(111): Development, Assessment, and Comparison. J Chem Theory Comput. 2018;14(6):3238-51.

170. Duin van, Adri C. T, Bryantsev VS, Diallo MS, Goddard WA, Rahaman O, et al. Development and Validation of a ReaxFF Reactive Force Field for Cu Cation/Water Interactions and Copper Metal/Metal Oxide/Metal Hydroxide Condensed Phases. J Phys Chem A. 2010;114(35):95079514.

171. Cheng T, Xiao H, Goddard WA. Reaction Mechanisms for the Electrochemical Reduction of CO2 to $\mathrm{CO}$ and Formate on the $\mathrm{Cu}(100)$ Surface at $298 \mathrm{~K}$ from Quantum Mechanics Free Energy Calculations with Explicit Water. J Am Chem Soc. 2016;138(42):13802-5.

172. Cheng, Tao H, Fortunelli A, Goddard III WA. Reaction intermediates during operando electrocatalysis identified from full solvent quantum mechanics molecular dynamics. Proc Natl Acad Sci. 2019;116(16):7718-22.

173. Clabaut P, Fleurat-Lessard P, Michel C, Steinmann SN. Ten Facets, One Force Field: The GAL19 Force Field for Water-Noble Metal Interfaces. J Chem Theory Comput [Internet]. 2020 [cited 2020]; Available from: https://doi.org/10.1021/acs.jctc.0c00091

174. Cramer CJ, Truhlar DG. Implicit Solvation Models: Equilibria, Structure, Spectra, and Dynamics. Chem Rev. 1999;99(8):2161-2200.

175. Fattebert J-L, Gygi F. First-principles molecular dynamics simulations in a continuum solvent. Int J Quantum Chem. 2003;93(2):139-147.

176. Ramirez R, Borgis D. Density Functional Theory of Solvation and Its Relation to Implicit Solvent Models. J Phys Chem B. 2005;109(14):6754-63.

177. Jeanmairet $G$, Levesque $M$, Vuilleumier R, Borgis D. Molecular Density Functional Theory of Water. J Phys Chem Lett. 2013;4(4):619-24.

178. Réocreux R, Jiang T, lannuzzi M, Michel C, Sautet P. Structuration and Dynamics of Interfacial Liquid Water at Hydrated $\mathrm{Y}$-Alumina Determined by ab Initio Molecular Simulations: Implications for Nanoparticle Stability. ACS Appl Nano Mater. 2018;1(1):191-9.

179. Limmer DT, Willard AP, Madden P, Chandler D. Hydration of metal surfaces can be dynamically heterogeneous and hydrophobic. Proc Natl Acad Sci U A. 2013;110(11):4200. 
180. Gauthier JA, Ringe S, Dickens CF, Garza AJ, Bell AT, Head-Gordon M, et al. Challenges in Modeling Electrochemical Reaction Energetics with Polarizable Continuum Models. ACS Catal. 2019;9(2):920-31.

181. Sun G, Sautet P. Metastable Structures in Cluster Catalysis from First-Principles: Structural Ensemble in Reaction Conditions and Metastability Triggered Reactivity. J Am Chem Soc. 2018;140(8):2812-20.

182. Marcus RA. On the Theory of Oxidation-Reduction Reactions Involving Electron Transfer. I. J Chem Phys. 1956;24(5):966-978.

183. Staub R, Steinmann SN. Parameter-free coordination numbers for solutions and interfaces. J Chem Phys. 2020;152(2):024124.

184. Sundararaman $R$, Goddard WA. The charge-asymmetric nonlocally determined local-electric (CANDLE) solvation model. J Chem Phys. 2015;142(6):064107.

185. Garcia-Ratés M, López N. Multigrid-Based Methodology for Implicit Solvation Models in Periodic DFT. J Chem Theory Comput. 2016;12(3):1331-41.

186. Fisicaro G, Genovese L, Andreussi O, Mandal S, Nair NN, Marzari N, et al. Soft-Sphere Continuum Solvation in Electronic-Structure Calculations. J Chem Theory Comput. 2017;13(8):3829-45.

187. Li Q, García-Muelas R, López N. Microkinetics of alcohol reforming for H2 production from a FAIR density functional theory database. Nat Commun. 2018;9(1):526.

188. Desai SK, Pallassana V, Neurock M. A Periodic Density Functional Theory Analysis of the Effect of Water Molecules on Deprotonation of Acetic Acid over Pd(111). J Phys Chem B. 2001;105(38):9171-9182.

189. Vatti AK, Todorova M, Neugebauer J. Ab Initio Determined Phase Diagram of Clean and Solvated Muscovite Mica Surfaces. Langmuir. 2016;32(4):1027-33.

190. Andreussi O, Hörmann NG, Nattino F, Fisicaro G, Goedecker S, Marzari N. Solvent-Aware Interfaces in Continuum Solvation. J Chem Theory Comput. 2019;15(3):1996-2009.

191. Moine E, Privat R, Sirjean B, Jaubert J-N. Estimation of Solvation Quantities from Experimental Thermodynamic Data: Development of the Comprehensive CompSol Databank for Pure and Mixed Solutes. J Phys Chem Ref Data. 2017;46(3):033102.

192. Andreussi O, Dabo I, Marzari N. Revised self-consistent continuum solvation in electronicstructure calculations. J Chem Phys. 2012;136(6):064102.

193. Sundararaman R, Schwarz KA, Letchworth-Weaver K, Arias TA. Spicing up continuum solvation models with SaLSA: The spherically averaged liquid susceptibility ansatz. J Chem Phys. 2015;142(5):054102.

194. Ringe S, Oberhofer H, Reuter K. Transferable ionic parameters for first-principles PoissonBoltzmann solvation calculations: Neutral solutes in aqueous monovalent salt solutions. J Chem Phys. 2017;146(13):134103. 
195. Truscott M, Andreussi O. Field-Aware Interfaces in Continuum Solvation. J Phys Chem B. 2019;123(16):3513-24.

196. Steinmann SN, Sautet $P$, Michel C. Solvation free energies for periodic surfaces: comparison of implicit and explicit solvation models. Phys Chem Chem Phys. 2016;18(46):31850-31861.

197. Akinola J, Barth I, Goldsmith BR, Singh N. Adsorption Energies of Oxygenated Aromatics and Organics on Rhodium and Platinum in Aqueous Phase. ACS Catal. 2020;4929-41.

198. Møller C, Plesset MS. Note on an Approximation Treatment for Many-Electron Systems. Phys Rev. 1934;46(7):618-622.

199. VandeVondele J, Hutter J. An efficient orbital transformation method for electronic structure calculations. J Chem Phys. 2003;118(10):4365-4369.

200. Mohr S, Ratcliff LE, Boulanger P, Genovese L, Caliste D, Deutsch T, et al. Daubechies wavelets for linear scaling density functional theory. J Chem Phys. 2014;140(20):204110.

201. Kohn W, Sham U. Self-Consistent Equations Including Exchange and Correlation Effects. Phys Rev. 1965;140(4A):A1133-8.

202. Kühne TD. Second generation Car-Parrinello molecular dynamics. Wiley Interdiscip Rev Comput Mol Sci. 2014;4(4):391-406.

203. Lin L, Chen M, Yang C, He L. Accelerating atomic orbital-based electronic structure calculation via pole expansion and selected inversion. J Phys Condens Matter. 2013;25(29):295501.

204. Mohr S, Eixarch M, Amsler M, Mantsinen MJ, Genovese L. Linear scaling DFT calculations for large tungsten systems using an optimized local basis. Nucl Mater Energy. 2018;64.

205. Hu Q-M, Reuter K, Scheffler M. Towards an Exact Treatment of Exchange and Correlation in Materials: Application to the "CO Adsorption Puzzle" and Other Systems. Phys Rev Lett. 2007;98(17):176103.

206. Gautier S, Steinmann SN, Michel C, Fleurat-Lessard P, Sautet P. Molecular adsorption at Pt(111). How accurate are DFT functionals? Phys Chem Chem Phys. 2015;17(43):2892128930.

207. Goerigk L, Grimme S. A General Database for Main Group Thermochemistry, Kinetics, and Noncovalent Interactions - Assessment of Common and Reparameterized (meta-)GGA Density Functionals. J Chem Theory Comput. 2010;6(1):107-26.

208. Steinmann SN, Corminboeuf C. Comprehensive Benchmarking of a Density-Dependent Dispersion Correction. J Chem Theory Comput. 2011;7(11):3567-77.

209. Perdew JP, Burke K, Ernzerhof M. Generalized Gradient Approximation Made Simple. Phys Rev Lett. 1996;77(18):3865-8.

210. Zhang Y, Yang W. Comment on "Generalized Gradient Approximation Made Simple." Phys Rev Lett. 1998;80(4):890.

211. Hammer B, Hansen LB, Norskov JK. Improved adsorption energetics within density-functional theory using revised Perdew-Burke-Ernzerhof functionals. Phys Rev B. 1999;59(11):7413. 
212. Perdew JP, Ruzsinszky A, Csonka GI, Vydrov OA, Scuseria GE, Constantin LA, et al. Restoring the Density-Gradient Expansion for Exchange in Solids and Surfaces. Phys Rev Lett. 2008;100(13):136406.

213. Gillan MJ, Alfè D, Michaelides A. Perspective: How good is DFT for water? J Chem Phys. 2016;144(13):130901.

214. Chen Y, Huang Y, Cheng T, Goddard WA. Identifying Active Sites for CO2 Reduction on Dealloyed Gold Surfaces by Combining Machine Learning with Multiscale Simulations. J Am Chem Soc. 2019;141(29):11651-11657.

215. Schmidt PS, Thygesen KS. Benchmark Database of Transition Metal Surface and Adsorption Energies from Many-Body Perturbation Theory. J Phys Chem C. 2018;122(8):4381-90.

216. Goerigk L, Hansen A, Bauer C, Ehrlich S, Najibi A, Grimme S. A look at the density functional theory zoo with the advanced GMTKN55 database for general main group thermochemistry, kinetics and noncovalent interactions. Phys Chem Chem Phys. 2017;19(48):32184-215.

217. Cohen AJ, Mori-Sanchez P, Yang W. Insights into Current Limitations of Density Functional Theory. Science. 2008;321(5890):792-4.

218. Ruzsinszky A, Perdew JP, Csonka GI, Vydrov OA, Scuseria GE. Spurious fractional charge on dissociated atoms: Pervasive and resilient self-interaction error of common density functionals. J Chem Phys. 2006;125(19):194112.

219. Patra A, Bates JE, Sun J, Perdew JP. Properties of real metallic surfaces: Effects of density functional semilocality and van der Waals nonlocality. Proc Natl Acad Sci. 2017;114(44):E9188-96.

220. Le Bahers T, Rérat $M$, Sautet $P$. Semiconductors Used in Photovoltaic and Photocatalytic Devices: Assessing Fundamental Properties from DFT. J Phys Chem C. 2014;118(12):59976008.

221. Bjorketun ME, Zeng Z, Ahmed R, Tripkovic V, Thygesen KS, Rossmeisl J. Avoiding pitfalls in the modeling of electrochemical interfaces. Chem Phys Lett. 2013;555(0):145-148.

222. Chu S, Majumdar A. Opportunities and challenges for a sustainable energy future. Nature. 2012;488(7411):294-303.

223. Khan K, Tareen AK, Aslam M, Zhang Y, Wang R, Ouyang Z, et al. Recent advances in twodimensional materials and their nanocomposites in sustainable energy conversion applications. Nanoscale. 2019;11(45):21622-78.

224. Lindgren $P$, Kastlunger $G$, Peterson AA. A Challenge to the $G \sim 0$ Interpretation of Hydrogen Evolution. ACS Catal. 2020;10(1):121-8.

225. Hinnemann B, Moses PG, Bonde J, Jørgensen KP, Nielsen JH, Horch S, et al. Biomimetic Hydrogen Evolution: MoS2 Nanoparticles as Catalyst for Hydrogen Evolution. J Am Chem Soc. 2005;127(15):5308-9.

226. Jaramillo TF, Jørgensen KP, Bonde J, Nielsen JH, Horch S, Chorkendorff I. Identification of Active Edge Sites for Electrochemical H2 Evolution from MoS2 Nanocatalysts. Science.

2007;317(5834):100-2. 
227. Benck JD, Chen Z, Kuritzky LY, Forman AJ, Jaramillo TF. Amorphous Molybdenum Sulfide Catalysts for Electrochemical Hydrogen Production: Insights into the Origin of their Catalytic Activity. ACS Catal. 2012;2(9):1916-23.

228. Kibsgaard J, Chen Z, Reinecke BN, Jaramillo TF. Engineering the surface structure of MoS 2 to preferentially expose active edge sites for electrocatalysis. Nat Mater. 2012;11(11):963-9.

229. Li H, Tsai C, Koh AL, Cai L, Contryman AW, Fragapane AH, et al. Activating and optimizing MoS 2 basal planes for hydrogen evolution through the formation of strained sulphur vacancies. Nat Mater. 2016;15(1):48-53.

230. Voiry D, Yang J, Chhowalla M. Recent Strategies for Improving the Catalytic Activity of 2D TMD Nanosheets Toward the Hydrogen Evolution Reaction. Adv Mater. 2016;28(29):6197-206.

231. Voiry D, Shin HS, Loh KP, Chhowalla M. Low-dimensional catalysts for hydrogen evolution and CO 2 reduction. Nat Rev Chem. 2018;2(1):1-17.

232. Voiry D, Salehi M, Silva R, Fujita T, Chen M, Asefa T, et al. Conducting MoS2 Nanosheets as Catalysts for Hydrogen Evolution Reaction. Nano Lett. 2013;13(12):6222-7.

233. Lukowski MA, Daniel AS, Meng F, Forticaux A, Li L, Jin S. Enhanced Hydrogen Evolution Catalysis from Chemically Exfoliated Metallic MoS2 Nanosheets. J Am Chem Soc. 2013;135(28):102747.

234. Voiry D, Yamaguchi H, Li J, Silva R, Alves DCB, Fujita T, et al. Enhanced catalytic activity in strained chemically exfoliated WS 2 nanosheets for hydrogen evolution. Nat Mater. 2013;12(9):850-5.

235. Chhowalla M, Shin HS, Eda G, Li L-J, Loh KP, Zhang H. The chemistry of two-dimensional layered transition metal dichalcogenide nanosheets. Nat Chem. 2013;5(4):263-75.

236. Di J, Yan C, Handoko AD, Seh ZW, Li H, Liu Z. Ultrathin two-dimensional materials for photoand electrocatalytic hydrogen evolution. Mater Today. 2018;21(7):749-70.

237. Hantanasirisakul K, Gogotsi Y. Electronic and Optical Properties of 2D Transition Metal Carbides and Nitrides (MXenes). Adv Mater. 2018;30(52):1804779.

238. Anasori B, Lukatskaya MR, Gogotsi Y. 2D metal carbides and nitrides (MXenes) for energy storage. Nat Rev Mater. 2017;2(2):1-17.

239. Naguib M, Mochalin VN, Barsoum MW, Gogotsi Y. 25th Anniversary Article: MXenes: A New Family of Two-Dimensional Materials. Adv Mater. 2014;26(7):992-1005.

240. Gogotsi Y, Anasori B. The Rise of MXenes. ACS Nano. 2019;13(8):8491-4.

241. Deysher G, Shuck CE, Hantanasirisakul K, Frey NC, Foucher AC, Maleski K, et al. Synthesis of Mo4VAIC4 MAX Phase and Two-Dimensional Mo4VC4 MXene with Five Atomic Layers of Transition Metals. ACS Nano. 2020;14(1):204-17.

242. Halim J, Kota S, Lukatskaya MR, Naguib M, Zhao M-Q, Moon EJ, et al. Synthesis and Characterization of 2D Molybdenum Carbide (MXene). Adv Funct Mater. 2016;26(18):311827. 
243. Naguib M, Mashtalir O, Carle J, Presser V, Lu J, Hultman L, et al. Two-Dimensional Transition Metal Carbides. ACS Nano. 2012;6(2):1322-31.

244. Fredrickson KD, Anasori B, Seh ZW, Gogotsi Y, Vojvodic A. Effects of Applied Potential and Water Intercalation on the Surface Chemistry of Ti2C and Mo2C MXenes. J Phys Chem C. 2016;120(50):28432-40.

245. Pandey M, Thygesen KS. Two-Dimensional MXenes as Catalysts for Electrochemical Hydrogen Evolution: A Computational Screening Study. J Phys Chem C. 2017;121(25):13593-8.

246. Seh ZW, Fredrickson KD, Anasori B, Kibsgaard J, Strickler AL, Lukatskaya MR, et al. TwoDimensional Molybdenum Carbide (MXene) as an Efficient Electrocatalyst for Hydrogen Evolution. ACS Energy Lett. 2016;1(3):589-94.

247. Handoko AD, Fredrickson KD, Anasori B, Convey KW, Johnson LR, Gogotsi Y, et al. Tuning the Basal Plane Functionalization of Two-Dimensional Metal Carbides (MXenes) To Control Hydrogen Evolution Activity. ACS Appl Energy Mater. 2018;1(1):173-80.

248. Li P, Zhu J, Handoko AD, Zhang R, Wang H, Legut D, et al. High-throughput theoretical optimization of the hydrogen evolution reaction on MXenes by transition metal modification. J Mater Chem A. 2018;6(10):4271-8.

249. Kuznetsov DA, Chen Z, Kumar PV, Tsoukalou A, Kierzkowska A, Abdala PM, et al. Single Site Cobalt Substitution in 2D Molybdenum Carbide (MXene) Enhances Catalytic Activity in the Hydrogen Evolution Reaction. J Am Chem Soc. 2019;141(44):17809-16.

250. Zhang J, Zhao Y, Guo X, Chen C, Dong C-L, Liu R-S, et al. Single platinum atoms immobilized on an $\mathrm{MXene}$ as an efficient catalyst for the hydrogen evolution reaction. Nat Catal. 2018;1(12):985-92.

251. Ramalingam V, Varadhan P, Fu H-C, Kim H, Zhang D, Chen S, et al. Heteroatom-Mediated Interactions between Ruthenium Single Atoms and an MXene Support for Efficient Hydrogen Evolution. Adv Mater. 2019;31(48):1903841.

252. Le TA, Bui QV, Tran NQ, Cho Y, Hong Y, Kawazoe Y, et al. Synergistic Effects of Nitrogen Doping on MXene for Enhancement of Hydrogen Evolution Reaction. ACS Sustain Chem Eng. 2019;7(19):16879-88.

253. Qu G, Zhou Y, Wu T, Zhao G, Li F, Kang Y, et al. Phosphorized MXene-Phase Molybdenum Carbide as an Earth-Abundant Hydrogen Evolution Electrocatalyst. ACS Appl Energy Mater. 2018;1(12):7206-12.

254. Li S, Tuo P, Xie J, Zhang X, Xu J, Bao J, et al. Ultrathin MXene nanosheets with rich fluorine termination groups realizing efficient electrocatalytic hydrogen evolution. Nano Energy. 2018;47:512-8.

255. Jiang $Y$, Sun T, Xie X, Jiang W, Li J, Tian B, et al. Oxygen-Functionalized Ultrathin Ti3C2Tx MXene for Enhanced Electrocatalytic Hydrogen Evolution. ChemSusChem. 2019;12(7):1368-73.

256. Zhan C, Sun W, Xie Y, Jiang D, Kent PRC. Computational Discovery and Design of MXenes for Energy Applications: Status, Successes, and Opportunities. ACS Appl Mater Interfaces. 2019;11(28):24885-905. 
257. Ling C, Shi L, Ouyang Y, Wang J. Searching for Highly Active Catalysts for Hydrogen Evolution Reaction Based on O-Terminated MXenes through a Simple Descriptor. Chem Mater. 2016;28(24):9026-32.

258. Ling C, Shi L, Ouyang Y, Chen Q, Wang J. Transition Metal-Promoted V2CO2 (MXenes): A New and Highly Active Catalyst for Hydrogen Evolution Reaction. Adv Sci. 2016;3(11):1600180.

259. Yang X, Gao N, Zhou S, Zhao J. MXene nanoribbons as electrocatalysts for the hydrogen evolution reaction with fast kinetics. Phys Chem Chem Phys. 2018;20(29):19390-7.

260. Rossmeisl J, Chan K, Skúlason E, Björketun ME, Tripkovic V. On the pH dependence of electrochemical proton transfer barriers. Catal Today. 2016;262:36-40.

261. McCrum IT, Janik MJ. pH and Alkali Cation Effects on the Pt Cyclic Voltammogram Explained Using Density Functional Theory. J Phys Chem C. 2016;120(1):457-71.

262. Ledezma-Yanez I, Wallace WDZ, Sebastián-Pascual P, Climent V, Feliu JM, Koper MTM. Interfacial water reorganization as a $\mathrm{pH}$-dependent descriptor of the hydrogen evolution rate on platinum electrodes. Nat Energy. 2017;2(4):17031.

263. Cheng T, Wang L, Merinov BV, Goddard WA. Explanation of Dramatic pH-Dependence of Hydrogen Binding on Noble Metal Electrode: Greatly Weakened Water Adsorption at High pH. J Am Chem Soc. 2018;140(25):7787-7790.

264. Lamoureux PS, Singh AR, Chan K. pH Effects on Hydrogen Evolution and Oxidation over Pt(111): Insights from First-Principles. ACS Catal. 2019;9(7):6194-201.

265. Liu L, Liu Y, Liu C. Enhancing the Understanding of Hydrogen Evolution and Oxidation Reactions on $\mathrm{Pt}(111)$ through $\mathrm{Ab}$ Initio Simulation of Electrode/Electrolyte Kinetics. J Am Chem Soc. 2020;142(11):4985-9.

266. Handoko AD, Wei F, Jenndy, Yeo BS, Seh ZW. Understanding heterogeneous electrocatalytic carbon dioxide reduction through operando techniques. Nat Catal. 2018;1(12):922-34.

267. Handoko AD, Khoo KH, Tan TL, Jin H, Seh ZW. Establishing new scaling relations on twodimensional MXenes for CO2 electroreduction. J Mater Chem A. 2018;6(44):21885-90.

268. Chen H, Handoko AD, Xiao J, Feng X, Fan Y, Wang T, et al. Catalytic Effect on CO2 Electroreduction by Hydroxyl-Terminated Two-Dimensional MXenes. ACS Appl Mater Interfaces. 2019;11(40):36571-9.

269. Lai Y, Jones RJR, Wang Y, Zhou L, Gregoire JM. Scanning Electrochemical Flow Cell with Online Mass Spectroscopy for Accelerated Screening of Carbon Dioxide Reduction Electrocatalysts. ACS Comb Sci. 2019;21(10):692-704.

270. Magnussen OM, Hotlos J, Nichols RJ, Kolb DM, Behm RJ. Atomic structure of Cu adlayers on $\mathrm{Au}(100)$ and $\mathrm{Au}(111)$ electrodes observed by in situ scanning tunneling microscopy. Phys Rev Lett. 1990;64(24):2929-2932.

271. Cai W-B, Wan L-J, Noda H, Hibino Y, Ataka K, Osawa M. Orientational Phase Transition in a Pyridine Adlayer on Gold(111) in Aqueous Solution Studied by in Situ Infrared Spectroscopy and Scanning Tunneling Microscopy. Langmuir. 1998;14(111):6992-6998. 
272. Khairunnisa A, Liao W, Yau S. Adsorption and Electrochemical Polymerization of Pyrrole on $\mathrm{Au}(100)$ Electrodes as Examined by In Situ Scanning Tunneling Microscopy. J Phys Chem C. 2016;120(46):26425-34.

273. Vu T-H, Wandlowski T. CV and in situ STM study the adsorption behavior of benzoic acid at the electrified $\mathrm{Au}(100) \mid \mathrm{HClO} 4$ interface: Structure and dynamics. J Electroanal Chem. 2016;776:40-8.

274. Vu T-H, Wandlowski T. Self-Assembled Structures of Benzoic Acid on Au(111) Surface. J Electron Mater. 2017;46(6):3463-71.

275. Lelaidier T, Leoni T, Ranguis A, D'Aléo A, Fages F, Becker C. Adsorption and Growth of Bispyrene Molecular Layers on Au(111) Studied by STM. J Phys Chem C. 2017;121(13):7214-20.

276. Monyoncho EA, Steinmann SN, Michel C, Baranova EA, Woo TK, Sautet P. Ethanol Electrooxidation on Palladium Revisited Using Polarization Modulation Infrared Reflection Absorption Spectroscopy (PM-IRRAS) and Density Functional Theory (DFT): Why Is It Difficult To Break the C-C Bond? ACS Catal. 2016;6(8):4894-4906.

277. Steinmann SN, Wei Z-Y, Sautet P. Theory and experiments join forces to characterize the electrocatalytic interface. Proc Natl Acad Sci. 2019;116(16):7611.

278. Fang $Y$, Ding $S$, Zhang M, Steinmann SN, Hu R, Mao B, et al. Revisiting the atomistic structures at the interface of $\mathrm{Au}(111)$ electrode-sulfuric acid solution. J Am Chem Soc. 2020;142:94399446.

279. Valette G. Double layer on silver single crystal electrodes in contact with electrolytes having anions which are slightly specifically adsorbed: Part III. The (111) face. J Electroanal Chem Interfacial Electrochem. 1989;269(1):191-203.

280. Sundararaman R, Schwarz K. Evaluating continuum solvation models for the electrodeelectrolyte interface: Challenges and strategies for improvement. J Chem Phys. 2017;146(8):084111.

281. Sottomayor MJ, Coelho V, Ferreira AP, Silva F, Baturina OA, Safonov VA, et al. Parameters of nhexanol adsorption on $\mathrm{Au}$ (111). Comparison between differential capacity and chronocoulometry results. Electrochimica Acta. 1999;45(4):775-87.

282. Foresti ML, Innocenti M, Guidelli R, Hamelin A. Electrochemical investigation of 1,5pentanediol adsorption on the $\mathrm{Ag}(111)$ and $\mathrm{Ag}(110)$ faces. J Electroanal Chem. 1999;467(1):217-29.

283. Chen A, Shi Z, Bizzotto D, Lipkowski J, Pettinger B, Bilger C. lodide adsorption at the Au(111) electrode surface. J Electroanal Chem. 1999;467(1):342-53.

284. Garcia-Araez N, Climent V, Herrero E, Feliu J, Lipkowski J. Thermodynamic studies of bromide adsorption at the $\mathrm{Pt}(111)$ electrode surface perchloric acid solutions: Comparison with other anions. J Electroanal Chem. 2006;591(2):149-58.

285. Shi Z, Lipkowski J, Mirwald S, Pettinger B. Electrochemical and second harmonic generation study of SO2-4 adsorption at the Au(111) electrode. J Electroanal Chem. 1995;396(1):115-24. 
286. Herrero E, Mostany J, Feliu JM, Lipkowski J. Thermodynamic studies of anion adsorption at the $\mathrm{Pt}(111)$ electrode surface in sulfuric acid solutions. J Electroanal Chem. 2002;534(1):79-89.

287. Mostany J, Martínez P, Climent V, Herrero E, Feliu JM. Thermodynamic studies of phosphate adsorption on $\mathrm{Pt}(111)$ electrode surfaces in perchloric acid solutions. Electrochimica Acta. 2009;54(24):5836-43.

288. Wandlowski Th, Hölzle MH. Structural and Thermodynamic Aspects of Phase Transitions in Uracil Adlayers. A Chronocoulometric Study. Langmuir. 1996;12(26):6604-15.

289. Li H-Q, Roscoe SG, Lipkowski J. Electrochemical Studies of the Benzoate Adsorption on Au (111) Electrode. J Solut Chem. 2000;29(10):987-1005.

290. Schmickler W, Guidelli R. The partial charge transfer. Electrochimica Acta. 2014;127:489-505. 